TRANSACTIONS OF THE

AMERICAN MATHEMATICAL SOCIETY

Volume 358, Number 6 , Pages $2579-2610$

S 0002-9947(05)03772-4

Article electronically published on October 21, 2005

\title{
BICYCLIC ALGEBRAS OF PRIME EXPONENT OVER FUNCTION FIELDS
}

\author{
BORIS È. KUNYAVSKIĬ, LOUIS H. ROWEN, SERGEY V. TIKHONOV, \\ AND VYACHESLAV I. YANCHEVSKII
}

\begin{abstract}
We examine some properties of bicyclic algebras, i.e. the tensor product of two cyclic algebras, defined over a purely transcendental function field in one variable. We focus on the following problem: When does the set of local invariants of such an algebra coincide with the set of local invariants of some cyclic algebra? Although we show this is not always the case, we determine when it happens for the case where all degeneration points are defined over the ground field. Our main tool is Faddeev's theory. We also study a geometric counterpart of this problem (pencils of Severi-Brauer varieties with prescribed degeneration data).
\end{abstract}

\section{INTRODUCTION AND PRELIMINARIES}

The main object of the present paper is a central simple algebra $\mathcal{A}$ defined over a function field in one variable $F=K(t)$, where $K$ is a field of characteristic zero. The main question is: when does the set of local invariants of $\mathcal{A}$ coincide with the set of local invariants of some cyclic algebra (in particular, a quaternion algebra)? Let us make this more precise. For a closed point $x \in \mathbb{P}_{K}^{1}$ denote $\mathfrak{g}_{x}=\operatorname{Gal}(\bar{K} / K(x))$, where $\bar{K}$ stands for the algebraic closure of $K$.

Definition 1.1. Let $F=K(t)$. Let $\mathcal{A}$ be a central simple $F$-algebra, and let $x \in \mathbb{P}_{K}^{1}$ be a closed point. The residue of $\mathcal{A}$ at $x$ is the element $\partial_{x}(\mathcal{A}) \in H^{1}\left(\mathfrak{g}_{x}, \mathbb{Q} / \mathbb{Z}\right)$, where $\partial_{x}: \operatorname{Br}(F) \rightarrow H^{1}\left(\mathfrak{g}_{x}, \mathbb{Q} / \mathbb{Z}\right)$ is the residue map [31, Ch. II, App., §3]. We call the pair $\left(x, \partial_{x}(\mathcal{A})\right)$ with nonzero $\partial_{x}(\mathcal{A})$ a local invariant of $\mathcal{A}$ at $x$.

Remark 1.2. There are many ways to define the residue map, which differ only by change of sign. Since we are mainly interested in the vanishing (or nonvanishing) of $\partial_{x}(\mathcal{A})$, we can use any of these definitions.

The local invariants of $\mathcal{A}$ cannot be arbitrary, but must satisfy the Faddeev reciprocity law.

Received by the editors November 5, 2002 and, in revised form, June 21, 2004.

2000 Mathematics Subject Classification. Primary 16K20.

This research was supported by the Israel Science Foundation founded by the Israel Academy of Sciences and Humanities - Center of Excellence Program and by RTN Network HPRN-CT$2002-00287$.

The first author was partially supported by the Ministry of Absorption (Israel), the Minerva Foundation through the Emmy Noether Research Institute of Mathematics, and INTAS 00-566.

The third and the fourth authors were partially supported by the Fundamental Research Foundation of Belarus, TMR ERB FMRX CT-97-0107, and INTAS 99-081.

(C)2005 American Mathematical Society Reverts to public domain 28 years from publication 
Proposition 1.3 ([11, [31, Ch. II, App., §5]). Let $n$ be a positive integer. There is an exact sequence

$$
0 \rightarrow{ }_{n} \operatorname{Br}(K) \rightarrow{ }_{n} \operatorname{Br}(F) \stackrel{\oplus \partial_{x}}{\longrightarrow} \bigoplus_{x \in \mathbb{P}_{K}^{1}} H^{1}(K(x), \mathbb{Z} / n) \stackrel{\text { cor }}{\longrightarrow} H^{1}(K, \mathbb{Z} / n) \rightarrow 0 .
$$

(Here $x$ runs over closed points of $\mathbb{P}_{K}^{1}, H^{1}(L, \cdot)$ is a shortening for $H^{1}(G a l(\bar{L} / L), \cdot)$, and cor is the sum of corestriction homomorphisms.)

In the sequel, we usually assume that $K$ contains the $n$th roots of unity and often identify $H^{1}(K, \mathbb{Z} / n)$ with $K^{*} /\left(K^{*}\right)^{n}$.

Note that Faddeev's reciprocity law shows that if the finite local invariants of two algebras are the same, then their local invariants at infinity are also the same. More generally, if $\mathcal{A}_{1}$ and $\mathcal{A}_{2}$ agree at all but one closed rational point of ramification, then they agree at that point.

Furthermore, if $\mathcal{A}$ has local invariants of order dividing $n$, then $\mathcal{A}^{\otimes n}$ has no nontrivial local invariants, and thus $\mathcal{A}^{\otimes n}$ is isomorphic to some constant algebra $\mathcal{B} \otimes_{K} F$.

We will use this observation without further reference.

For a finite collection $\left\{x_{i}, d_{i}\right\}$, where $x_{i} \in \mathbb{P}_{K}^{1}$ is a closed point and $d_{i} \in$ $H^{1}\left(K\left(x_{i}\right), \mathbb{Z} / n\right)$, we denote by $\phi \in \bigoplus_{x \in \mathbb{P}_{K}^{1}} H^{1}(K(x), \mathbb{Z} / n)$ the element with coordinate $d_{i}$ at $x_{i}$ and zeros elsewhere. We say that the collection $\left\{x_{i}, d_{i}\right\}$ satisfies the Faddeev reciprocity law if $\phi \in \operatorname{ker}(\mathrm{cor})$. In this case we also say $\left\{x_{i}, d_{i}\right\}$ is a system of local invariants of order dividing $n$ (and sometimes even abusively shorten to speaking about invariants of order $n$ ). Our main question can be formulated as follows:

Question A. Given a finite collection $\left\{x_{i}, d_{i}\right\}$, where $x_{i} \in \mathbb{P}_{K}^{1}$ is a closed point and $d_{i} \in H^{1}\left(K\left(x_{i}\right), \mathbb{Z} / n\right)$, satisfying the Faddeev reciprocity law, does there exist a cyclic algebra $Q$ of degree $n$ over $F=K(t)$ which ramifies only at the $x_{i}$ 's, such that the residue of $Q$ at $x_{i}$ equals $d_{i}$ for each $i$ ?

The answer to Question A is negative in general, as we shall discuss below.

Let us define the exponent of $\mathcal{A}$ to be the order of the class of $\mathcal{A}$ in the Brauer group. If $K$ is finite (i.e. $F$ is a global field), the classical Albert-Brauer-HasseNoether theorem guarantees that any central simple algebra of exponent $n$ is isomorphic to a cyclic algebra of degree $n$. For more general $K$ such a fact may no longer hold, and obstructions may arise in various ways (see, for example, [10, [20], [7, [29]).

Any central simple algebra of exponent $n$ gives rise to its system of local invariants of order $n$.

Conversely by [11, for any system of local invariants $\left\{x_{i}, d_{i}\right\}$ having order $n$, there exists a central simple algebra $\mathcal{A}$ over $F$ with these local invariants, and $\mathcal{A}$ is determined uniquely up to tensor multiplication by a constant algebra (i.e. algebra coming from a $K$-algebra). We can always choose $\mathcal{A}$ to have exponent $n$ using classical results of Auslander-Brumer, Fein-Schacher and Rosset-Tate, guaranteeing the existence of an algebra of exponent $n$ with given invariants of order $n$ which is a tensor product of a limited number of cyclic algebras:

Proposition 1.4. Let $K$ be any field. Given a finite collection $\left\{v_{i}, d_{i}\right\}$, where $v_{i}$ is a discrete valuation of $F$ and $d_{i} \in H^{1}\left(K\left(v_{i}\right), \mathbb{Z} / n\right)$, satisfying the Faddeev reciprocity law, there exists an algebra $\mathcal{A}$ over $F=K(t)$ of exponent $n$ unramified 
outside the $v_{i}$ 's and such that the ramification of $\mathcal{A}$ at $v_{i}$ is $d_{i}$. In fact, if $K$ contains the nth roots of unity, $\mathcal{A}$ is a tensor product of $\leq \sum \operatorname{deg} f_{j}$ symbols each of degree $n$, where $f_{j}$ are the polynomials defining the finite valuations of the collection $\left\{v_{i}\right\}$.

Proof. According to [12, Prop. 1] (see [13, p. 254] for minor corrections), under hypotheses of the proposition, the Faddeev exact sequence splits as a sequence of abelian groups (this result goes back to [4, Prop. 4.1]). Hence there exists a Brauer class of order $n$. By 28, it is a product of the appropriate number of cyclic algebras of degree $n$.

In this way, we see that any system of local invariants of order $n$ corresponds to algebras of exponent $n$.

Definition 1.5. We say that a system of local invariants $\left\{x_{i}, d_{i}\right\}$ of order $n$ is good if it is realizable as the system of local invariants of an algebra of degree $n$, and bad otherwise.

Remark 1.6. For $n \leq 3$ all division algebras of degree $n$ are cyclic, but for $n$ prime $\geq 5$ this remains an open problem.

Our fundamental question of which systems are good leads us to the following concept.

Definition 1.7. Let $K$ be a field, $F=K(t)$. We call central simple $F$-algebras $\mathcal{A}$ and $\mathcal{B}$ Faddeev equivalent if there is a $K$-algebra $\mathcal{C}$ such that $\mathcal{A}$ is Brauer equivalent to $\mathcal{B} \otimes_{K} \mathcal{C}$. We define the Faddeev index of $\mathcal{A}$ as the minimum degree of algebras Faddeev equivalent to $\mathcal{A}$.

Note that the Faddeev index is a Brauer invariant since $\mathrm{M}_{n}(\mathcal{A}) \sim \mathrm{M}_{n}(K) \otimes_{K} \mathcal{A}$ is Faddeev equivalent to $\mathcal{A}$. Thus the Faddeev index of an algebra $\mathcal{A}$ of exponent $n$ equals $n$ if and only if the collection of local invariants of $\mathcal{A}$ is good.

Problem B. Compute the Faddeev index of any given $\mathcal{A}$.

In Section 2 we consider the case of an arbitrary ground field $K$. We focus on an important particular case where all $x_{i}$ 's are defined over $K$. As a corollary, we obtain an alternative proof of the results of Ford, Van den Bergh and Jacob on cyclicity of $k(x, y)$-algebras ramified along a cubic or a hyperelliptic curve ( $k$ is an algebraically closed field). We thank the referee for bringing this application to our attention. For the reader's convenience, we discuss this matter in the Appendix (see Theorems $\mathrm{A}[5$ and $\mathrm{A}(6) 11$ Another goal of Section 2 is to find effective criteria for a system of local invariants to be good.

Although Problem B seems to be out of reach for general $K$, for some fields the situation looks more optimistic. Namely, if $K$ is a $\mathfrak{p}$-adic field (= a finite extension of $\mathbb{Q}_{p}$ ), the main result of Saltman's paper [29] gives a bound for the index of algebra of fixed exponent over the function field of a curve defined over $K$. If $\left[K: \mathbb{Q}_{p}\right]<\infty$, by [29, Th. 3.4], the index of any $K(t)$-algebra $\mathcal{A}$ of exponent $n$ prime to $p$ divides $n^{2}$. Moreover, the appendix to 29] contains an example (due to Jacob and Tignol) showing that this estimate is sharp: The Jacob-Tignol algebra has exponent 2 and index 4. Thus, in order to describe all $K(t)$-algebras of prime exponent, without loss of generality one can focus on bicyclic algebras and look for a criterion for

\footnotetext{
${ }^{1}$ Added in proof. Our paper Division algebras that ramify only on a plane quartic curve (to appear in Proc. Amer. Math. Soc.) contains more general results.
} 
such an algebra to have index $p^{2}$. In a particular case, such a criterion is given in Proposition 3.15

The Jacob-Tignol algebra turns out to be Faddeev equivalent to a quaternion algebra (see Remark 3.4 below) and thus has Faddeev index 2. Therefore our first main goal is to find examples of bad local invariants in the case of algebras defined over $K(t),\left[K: \mathbb{Q}_{p}\right]<\infty$. An abundance of such examples can be found in Section 3 (see Theorem 3.6).

In Section 4 we consider the case where $K$ is a number field focusing on "Hasse principles".

Our paper also has a geometric aspect, and in fact our primary motivation came from a geometric problem where $n=2$. Let $X$ be a conic bundle rational surface defined over a field $K$. This means that there is a dominant morphism $\varphi: X \rightarrow \mathbb{P}_{K}^{1}$ with generic fiber isomorphic to a smooth conic. Such a fibration degenerates at a finite number of closed points $x_{i} \in \mathbb{P}_{K}^{1}$, and each degenerate fiber consists of a pair of smooth rational curves transversally intersecting at one point [16, 17]. Assume that $\varphi$ is relatively minimal, i.e. no degenerate fiber can be blown down. Then each component of such a fiber is defined over a quadratic extension $L_{i}$ of the residue field $K\left(x_{i}\right)$.

Definition 1.8. Let $\varphi: X \rightarrow \mathbb{P}_{K}^{1}$ be a relatively minimal conic bundle surface. The set of local invariants is defined as the collection of quadratic extensions $\left\{L_{i} / K\left(x_{i}\right)\right\}$, where $x_{i} \in \mathbb{P}_{K}^{1}$ are closed points at which $\varphi$ degenerates, $K\left(x_{i}\right)$ is the residue field of $x_{i}$, and $L_{i}$ is the field of definition of the components of the degenerate fiber at $x_{i}$.

We would like to describe $X$ in terms of this local information. The first question is existence:

Question C. Given a finite set $\left\{K\left(x_{i}\right), L_{i}\right\}$, where $x_{i} \in \mathbb{P}_{K}^{1}$ is a closed point and $L_{i}$ is a quadratic extension of $K\left(x_{i}\right)$, does there exist a (relatively minimal) conic bundle $\varphi: X \rightarrow \mathbb{P}_{K}^{1}$ with local invariants $\left\{K\left(x_{i}\right), L_{i}\right\}$ ?

A natural obstruction to a positive answer to Question $\mathrm{C}$ becomes evident as soon as this question is translated into the language of quaternion algebras. Indeed, let $Q$ denote a quaternion algebra over $F=K(t)$ corresponding to the generic fiber of $X$. Then $Q$ ramifies precisely at the $x_{i}$ 's, and its residue at $x_{i}$ can be identified with a nonzero element of $H^{1}\left(K\left(x_{i}\right), \mathbb{Z} / 2\right)=K\left(x_{i}\right)^{*} /\left(K\left(x_{i}\right)^{*}\right)^{2}$ which, in turn, corresponds to some quadratic extension $L_{i} / K\left(x_{i}\right)$. More precisely, we have

Proposition 1.9 ([16, [17, 7]). (i) There is a one-to-one correspondence between classes of birational (fiber-preserving) isomorphisms of relatively minimal conic bundles $\varphi: X \rightarrow \mathbb{P}_{K}^{1}$ and isomorphism classes of quaternion algebras over $F=K(t)$.

(ii) Let $x \in \mathbb{P}_{K}^{1}$ be a closed point. There is a one-to-one correspondence between the following data:

- collection of quadratic and trivial extensions of $K(x)$;

- $H^{1}\left(\mathfrak{g}_{x}, \mathbb{Z} / 2\right)$;

- $K(x)^{*} /\left(K(x)^{*}\right)^{2}$.

(iii) There is a one-to-one correspondence between closed points of $\mathbb{P}_{K}^{1}$ and discrete valuations of $K(t)$ trivial on $K$. 
We thus get a one-to-one correspondence between the set of local invariants of a conic bundle $\varphi: X \rightarrow \mathbb{P}_{K}^{1}$ and the set of local invariants of the corresponding quaternion algebra.

Hence, even if a given set $\left(K\left(x_{i}\right), L_{i}\right)$ of local invariants satisfies Faddeev's reciprocity law (which is an obvious necessary condition for the positive answer to Question C), it may not correspond to any conic bundle surface, being bad in the sense of Definition 1.5.

We also consider a generalization of Question C, with quaternion algebras replaced by cyclic algebras of odd prime exponent. Suitable geometric objects (pencils of cyclic Severi-Brauer varieties) are studied in Section 5. Apart from an almost straightforward translation of results of Section 3 into geometric language, we present another setting of the above questions. Namely, it turns out that if we are allowed to move the points of degeneration $x_{i}$, then the answer to the corresponding questions will always be positive (see Theorem [5.10). For the conic bundle case such a setting was considered in 21]. These results can be viewed as an analog of Manin's problem 25]: to construct a rational surface $X$ with the prescribed action of $\operatorname{Gal}(\bar{K} / K)$ on the Picard group $\operatorname{Pic}\left(X \times_{K} \bar{K}\right)$.

Notation, conventions, and background material. $K$ will always denote a ground field of characteristic 0 . It is often a p-adic field (i.e. a finite extension of $\mathbb{Q}_{p}$, the field of $p$-adic numbers). When that is the case, $R$ is the ring of integers of $K$, and we fix a uniformizer $\pi$ once and for all. Then $\mathfrak{p}=(\pi)$ is the unique maximal ideal of $R$, and $k=R / \mathfrak{p}$ will denote the residue field. For any polynomial $\gamma \in R[t]$ we designate its reduction modulo $\mathfrak{p}$ by $\bar{\gamma} \in k[t]$. We fix an integer $n$. (Our motivating questions were for $n=2$.) We usually assume that $R$ contains an $n$th root of unity $\rho$ and fix $\rho$ once and for all. (Note that this assumption might be very important for problems under consideration; cf. 6].) We always assume that $\operatorname{char}(k)$ is prime to $n$.

We denote by $\operatorname{Br}(T)$ the Brauer group of a ring $T$. If $a, b \in T^{*}$, we denote by $(a, b)_{n}$ the cyclic algebra generated over $T$ by elements $\alpha, \beta$ such that $\alpha^{n}=a$, $\beta^{n}=b, \alpha \beta=\rho \beta \alpha$ (we often drop the subscript $n$ if this does not lead to any confusion). If $\mathcal{A}$ is a central simple algebra, we denote by $\mathcal{A}^{\mathrm{op}}$ the opposite algebra (whose Brauer class is inverse to the Brauer class of $\mathcal{A}$ ).

Let $F$ be a field with discrete valuation $v$, let $\kappa$ be the residue field, let $z$ be a uniformizing element and let $\mathcal{A}$ be a central simple $F$-algebra of exponent $n$. In the equicharacteristic case (i.e. if $\operatorname{char}(F)=\operatorname{char}(\kappa)$ ), the completion of $F$ with respect to $v$ is of the form $\kappa((z))$. Then

$$
\mathcal{A} \otimes_{F} \kappa((z)) \sim\left(\mathcal{B} \otimes_{\kappa} \kappa((z))\right) \otimes(u, z)_{n}
$$

where $u \in \kappa$ and $\mathcal{B}$ is a $\kappa$-algebra (see [35]). Then the ramification of $\mathcal{A}$ at $v$ is $\kappa(\sqrt[n]{u}) / \kappa$. Note that the ramification of $(a, b)_{n}$ is defined by the $n$th root of the residue of $(-1)^{v(a) v(b)} a^{v(b)} / b^{v(a)}$ (see, for example, [5], 29]).

If $G$ is an (additively written) abelian group and $n: G \rightarrow G$ is multiplication by $n$, then ${ }_{n} G$ (resp. $G / n$ ) stands for the kernel (resp. cokernel) of $n$.

We use the notation $\left\langle a_{1}, \ldots, a_{m}\right\rangle$ for the diagonal quadratic form $a_{1} x_{1}^{2}+\cdots+$ $a_{m} x_{m}^{2}$, and $\langle c\rangle \cdot\left\langle a_{1}, \ldots, a_{m}\right\rangle$ for $c a_{1} x_{1}^{2}+\cdots+c a_{m} x_{m}^{2}$.

Other notation will be explained when needed. 
Recall the structure of discrete valuations of $F=K(t)$ which are trivial on $K$. There are two possibilities: If the valuation corresponds to a finite closed point $x$ of $\mathbb{P}_{K}^{1}$, then there exists an irreducible monic polynomial $f(t) \in K[t]$ such that the valuation $v_{f}$ with $f(t)$ as a uniformizer coincides with the valuation corresponding to $x$. The valuation corresponding to the infinite point is $v_{\infty}$ with $t^{-1}$ as a uniformizer. The completions of $K(t)$ with respect to $v_{f}$ and $v_{\infty}$ are of the form $K(\theta)((f(t)))$ and $K\left(\left(t^{-1}\right)\right)$, respectively. Here $\theta$ is a root of $f(t)$, and the embedding $K(t) \hookrightarrow$ $K(\theta)((f(t)))$ sends $t$ to some series $\tilde{t}$ such that

$$
\tilde{t} \equiv \theta \quad(\bmod f(t)) .
$$

If $\mathcal{A}$ is a central simple $F$-algebra and $f(t) \in K[t]$ is a monic irreducible polynomial defining a valuation of $F$, we denote $\mathcal{A}_{f}=\mathcal{A} \otimes K(\theta)((f(t)))$. We shall denote $K_{v}$ as the residue field of the valuation $v$ of $F$.

Let $f(t), g(t) \in K[t]$, with $f$ irreducible and not dividing $g$. Then the ramification of $(g(t), f(t))_{n}$ at $f$ is $K(\theta)(\sqrt[n]{g(\theta)}) / K(\theta)$, where $\theta$ is a root of $f(t)$.

The following results on quadratic forms are very useful in the case $n=2$.

Theorem 1.10 (Albert, [1, Th. 3]). Let $\mathcal{A}=(a, b) \otimes(c, d)$ be a tensor product of quaternion algebras defined over an arbitrary field $F$. Then $\mathcal{A}$ is a division algebra if and only if the quadratic form in six variables $\langle a, b,-a b,-c,-d, c d\rangle$ is anisotropic over $F$.

Theorem 1.11 (Springer, 23, Ch. VI, Prop. 1.9]). Let $F$ be a field complete with respect to a valuation $v: F^{*} \rightarrow \mathbb{Z}$ having uniformizer $\pi$. Suppose that the characteristic of the residue field $\kappa$ is different from 2. Then any F-anisotropic form $q$ can be written uniquely as an orthogonal sum

$$
q=q_{1} \perp\langle\pi\rangle q_{2}=\left\langle u_{1}, \ldots, u_{r}\right\rangle \perp\langle\pi\rangle \cdot\left\langle u_{r+1}, \ldots, u_{n}\right\rangle
$$

with $u_{i}$ units, and the reduction $\bar{q}_{i}(i=1,2)$ anisotropic over $\kappa$. Conversely, given a representation of $q$ as above where $\bar{q}_{1}$ and $\bar{q}_{2}$ are anisotropic over $\kappa, q$ is anisotropic over $F$.

Remark 1.12. We shall apply Springer's theorem in the case where $F$ is not complete in the following way. With the notation of Theorem 1.11, if $\bar{q}_{1}$ and $\bar{q}_{2}$ are anisotropic over $k$, then $q$ is anisotropic over $F$.

The following result (close to that in [29, Claim, p. 44]) is a useful alternative to the Albert form and gives us a way of finding division algebras of index $p^{2}$ and exponent $p$. It often appears in the literature in slightly different forms (cf. [26], [19]). For the reader's convenience, we give a proof here.

Proposition 1.13. Let $S$ be a discrete valuation ring with field of fractions $F$, residue field $\kappa$, and uniformizer $\pi$. Assume $F$ and $\kappa$ have a primitive nth root of one. Let $u \in S^{*}$ with image $\bar{u} \in \kappa$ be such that $\kappa\left(\bar{u}^{1 / n}\right) / \kappa$ has degree $n$. Suppose $\mathcal{D} / F$ is a central simple algebra with order $\mathcal{A} / S$ such that $\mathcal{A} / S$ is an Azumaya algebra. Let $\overline{\mathcal{A}}=\mathcal{A} / \pi \mathcal{A}$ and assume $\overline{\mathcal{A}} \otimes_{\kappa} \kappa\left(\bar{u}^{1 / n}\right) / \kappa$ is a division algebra. Then $\mathcal{D} \otimes_{F}(\pi, u)_{n}$ is a division algebra.

Proof. In order to use Theorem 5.15 from [19, one has to go over to the henselization $F_{h}$ of the field $F$. We have $\bar{F}_{h}=\kappa$.

We need some more terminology and notation from [19. If $\mathcal{B}$ is a division algebra over a henselian field $F_{h}$ with a valuation $v$, and $\left[\mathcal{B}: F_{h}\right]<\infty$, then $v$ 
extends uniquely to a valuation of $\mathcal{B}$. We denote by $\Gamma_{\mathcal{B}}$ and $\Gamma_{F_{h}}$ the corresponding value groups. $V_{\mathcal{B}}$ denotes the valuation ring of the extended valuation (cf. [19, p. 130]).

Let us show that $\mathcal{D} \otimes_{F} F_{h}$ is unramified over $F_{h}$, and $(\pi, u)_{n} \otimes_{F} F_{h}$ is nicely semiramified (see [19, p. 149] for the definition). Let $\mathcal{D}_{h}=\mathcal{D} \otimes_{F} F_{h}$ and $\mathcal{A}_{h}=$ $\mathcal{A} \otimes_{S} S_{h}$, where $S_{h}$ denotes the valuation ring of $F_{h}$.

Since $\mathcal{A} / S$ is an Azumaya algebra, $\mathcal{A}_{h} / S_{h}$ is also an Azumaya algebra.

Furthermore, it immediately follows from the definition that $\mathcal{A}_{h}$ is an order in $\mathcal{D}_{h}$, in the sense that $\mathcal{A}_{h}$ is integral over $S_{h}$ and its ring of fractions contains $\mathcal{D}$ and $F_{h}$, and thus $\mathcal{D}_{h}$. This implies that $\overline{\mathcal{A}}_{h}$ is a central simple algebra over $\kappa$.

Note that $\overline{\mathcal{A}}=\overline{\mathcal{A}}_{h}$. Indeed, $\overline{\mathcal{A}}$ is a central simple subalgebra with

$$
[\overline{\mathcal{A}}: \kappa]=[\mathcal{A}: S]=[\mathcal{D}: F]=\left[\mathcal{A}_{h}: S_{h}\right]=\left[\overline{\mathcal{A}_{h}}: \kappa\right],
$$

implying $\overline{\mathcal{A}}=\overline{\mathcal{A}}_{h}$.

Since $\overline{\mathcal{A}} \otimes_{\kappa} \kappa\left(\bar{u}^{1 / n}\right) / \kappa$ is a division algebra, $\overline{\mathcal{A}}=\overline{\mathcal{A}}_{h}$ is also a division algebra.

Let us show that $\mathcal{D}_{h}$ is a division algebra over $F_{h}$. Suppose $\mathcal{D}_{h} \cong M_{r}\left(\mathcal{D}_{1}\right)$, where $\mathcal{D}_{1}$ is a division algebra over $F_{h}$. Since $F_{h}$ is henselian, one can extend the valuation from $F_{h}$ to $\mathcal{D}_{1}$. We denote the corresponding valuation ring by $V_{\mathcal{D}_{1}}$.

Since $\mathcal{A}_{h}$ is an order in $M_{r}\left(\mathcal{D}_{1}\right)$, Proposition 2.5 from [19] implies that $\mathcal{D}_{1}$ is unramified over $F_{h}$ and $\mathcal{A}_{h} \cong M_{r}\left(V_{\mathcal{D}_{1}}\right)$. Then $\overline{\mathcal{A}}_{h} \cong M_{r}\left(\overline{\mathcal{D}}_{1}\right)$, implying $r=1$. Thus $\mathcal{D}_{h}=\mathcal{D}_{1}$, i.e. $\mathcal{D}_{h}$ is a division algebra. Moreover, we have proved that $\mathcal{A}_{h}=V_{\mathcal{D}_{h}}$, i.e. $\overline{\mathcal{A}}_{h}=\overline{\mathcal{D}}_{h}$. Thus $\mathcal{D}_{h}$ is unramified over $F_{h}$.

By [19, Theorem 4.4(iii)], $(\pi, u)_{n} \otimes_{F} F_{h}$ is nicely semiramified. We can now use Theorem 5.15 of [19]. Note that $\overline{(\pi, u)_{n} \otimes_{F} F_{h}}=\overline{F_{h}\left(u^{1 / n}\right)}=\kappa\left(\bar{u}^{1 / n}\right)$ and $\left|\Gamma_{(\pi, u) \otimes F_{h}}: \Gamma_{F_{h}}\right|=n$ (see [19, Example 4.3]).

To show that $\mathcal{D}_{h} \otimes_{F_{h}}\left((\pi, u)_{n} \otimes_{F} F_{h}\right)=\left(\mathcal{D} \otimes_{F}(\pi, u)_{n}\right) \otimes_{F} F_{h}$ is a division algebra, it is enough to prove that its degree equals the index.

We have

$$
\begin{gathered}
\operatorname{deg}\left(\left(\mathcal{D}_{h} \otimes_{F}(\pi, u)_{n}\right) \otimes_{F} F_{h}\right)=\sqrt{\left[\left(\mathcal{D} \otimes_{F}(\pi, u)_{n}\right) \otimes_{F} F_{h}: F_{h}\right]} \\
=\sqrt{\left[\mathcal{D}_{h}: F_{h}\right]} \cdot \sqrt{\left[(\pi, u)_{n}: F\right]}=n \cdot \sqrt{\left[\mathcal{D}_{h}: F_{h}\right]} .
\end{gathered}
$$

On the other hand, from [19, Theorem 5.15(a)] it follows that

$$
\begin{aligned}
& \operatorname{ind}\left(\left(\mathcal{D}_{h} \otimes_{F}(\pi, u)_{n}\right) \otimes_{F} F_{h}\right)=\operatorname{ind}\left(\overline{\mathcal{D}}_{h} \otimes_{\kappa} \overline{(\pi, u)_{n} \otimes_{F} F_{h}}\right) \cdot\left|\Gamma_{(\pi, u) \otimes F_{h}}: \Gamma_{F}\right| \\
& \quad=\operatorname{ind}\left(\overline{\mathcal{D}}_{h} \otimes_{\kappa} \kappa\left(\bar{u}^{1 / n}\right)\right) \cdot\left|\Gamma_{(\pi, u) \otimes F_{h}}: \Gamma_{F}\right|=n \cdot \operatorname{ind}\left(\overline{\mathcal{D}}_{h} \otimes_{\kappa} \kappa\left(\bar{u}^{1 / n}\right)\right) .
\end{aligned}
$$

Since $\overline{\mathcal{D}}_{h} \otimes_{\kappa} \kappa\left(\bar{u}^{1 / n}\right)$ is a division algebra, its index equals the degree, i.e.

$$
\operatorname{ind}\left(\overline{\mathcal{D}}_{h} \otimes_{\kappa} \kappa\left(\bar{u}^{1 / n}\right)\right)=\sqrt{\left[\overline{\mathcal{D}}_{h} \otimes_{\kappa} \kappa\left(\bar{u}^{1 / n}\right): \kappa\left(\bar{u}^{1 / n}\right)\right]}=\sqrt{\left[\overline{\mathcal{D}}_{h}: \kappa\right]} .
$$

But $\left[\overline{\mathcal{D}}_{h}: \kappa\right]=\left[\mathcal{D}_{h}: F_{h}\right]\left(\mathcal{D}_{h}\right.$ is unramified over $\left.F\right)$. Hence

$$
\left.\operatorname{ind}\left(\left(\mathcal{D}_{h} \otimes_{F}(\pi, u)_{n}\right) \otimes_{F} F_{h}\right)=n \cdot \sqrt{\left[\mathcal{D}_{h}: F_{h}\right]}=\operatorname{deg}\left(\mathcal{D} \otimes_{F}(\pi, u)_{n}\right) \otimes_{F} F_{h}\right) .
$$

Thus $\left.\left(\mathcal{D} \otimes_{F}(\pi, u)_{n}\right) \otimes_{F} F_{h}\right)$ is a division algebra. We conclude that $\mathcal{D} \otimes_{F}(\pi, u)_{n}$ is a division algebra. 
Corollary 1.14. Let $\mathcal{A}=(\pi, g) \otimes\left(h_{1}, h_{2}\right)$ be a bicyclic algebra over $F=K(t)$, a product of two symbols of degree $n$. Denote by $v$ the valuation of $F$ obtained by extending the given discrete valuation of $K$ such that $v(t)=0$ and the residue field of $v$ is $\kappa=k(t))$, and assume

$$
v(g)=v\left(h_{1}\right)=v\left(h_{2}\right)=0 .
$$

Assume also that $\kappa\left(\bar{g}^{1 / n}\right) / \kappa$ has degree $n$.

$$
\text { Let }
$$

$$
\overline{\mathcal{A}}=\left(\bar{h}_{1}, \bar{h}_{2}\right) \otimes_{\kappa} \kappa(\sqrt[p]{\bar{g}}) .
$$

If $\overline{\mathcal{A}}$ is a division algebra, then $\mathcal{A}$ is a division algebra.

Proof. It is enough to note that the algebra $\left(h_{1}, h_{2}\right)$ has an order $\mathcal{C} / S$ such that $\mathcal{C} / S$ is an Azumaya algebra and $\overline{\mathcal{C}}=\left(\bar{h}_{1}, \bar{h}_{2}\right)$; cf. Example 2.4(i) of [19].

\section{Generalities OVER AN ARBitrary FiELD}

In this section we describe several classes of algebras Faddeev-similar to cyclic algebras. First, these are algebras with "small" ramification locus defined over the ground field (Corollary 2.4). Second, for $n=2$, we explicitly construct a large family of algebras of Faddeev index 2 (Propositions 2.8, 2.9). We assume $K$ to be an arbitrary field containing an $n$th root of unity.

We start with the special case for which all ramification points are defined over $K$.

Definition 2.1. If all ramification points of $\mathcal{A}$ are defined over $K$ we say that $\mathcal{A}$ has linear ramification.

Indeed, in that case all (finite) ramification points of $\mathcal{A}$ correspond to linear polynomials $t-x_{i}$.

Our first result concerns algebras ramified only at three linear points.

Proposition 2.2. Let $K$ be an arbitrary field, let $F=K(t)$, and let $\mathcal{A}$ be a central simple $F$-algebra of exponent $n$. Suppose that $\mathcal{A}$ has ramification at exactly three linear points. Then $\mathcal{A}$ is Faddeev equivalent to a cyclic F-algebra of degree $n$.

Proof. Using the automorphism group of $\mathbb{P}_{K}^{1}$ one may assume that the points of ramification of $\mathcal{A}$ are $t, t-1$ and $\infty$ with ramification $K\left(\sqrt[n]{b_{1}}\right) / K, K\left(\sqrt[n]{b_{2}}\right) / K$, and $K\left(\sqrt[n]{b_{3}}\right) / K$ respectively.

The cyclic algebra

$$
\mathcal{B}=\left(b_{2} t,-b_{1}^{-1}(t-1)\right)
$$

has the prescribed ramification at $t$ and $t-1$. Hence by the reciprocity law, $\mathcal{B}$ has ramification $K\left(\sqrt[n]{b_{3}}\right) / K$ at $\infty$. Thus $\mathcal{A}$ is Faddeev equivalent to $\mathcal{B}$.

We proceed to the case where $\mathcal{A}$ has a quadratic ramification point. We show that $\mathcal{A}$ is Faddeev equivalent to a cyclic algebra, even if it has one additional linear ramification point. We may (and shall) assume that this linear point is infinite.

Proposition 2.3. Let $\mathcal{A}$ be a central simple $F$-algebra of exponent $n$ whose finite ramification is only at a point corresponding to a monic quadratic irreducible polynomial $f$ with ramification given by a cyclic extension $K(\theta)(\sqrt[n]{u+v \theta}) / K(\theta)$, where $\theta$ is a root of $f, u, v \in K$, and perhaps with ramification at infinity. Then if $v=0$, $\mathcal{A}$ is Faddeev equivalent to $(f, u)_{n}$, and if $v \neq 0$, then $\mathcal{A}$ is Faddeev equivalent to $(u+v t, f(t) / f(-u / v))_{n}$. 
Proof. First suppose $v=0$. Consider the algebra $\mathcal{B}=(u, f)_{n}$. It is ramified at $f$ and maybe at infinity. Its ramification at $f$ coincides with the ramification of $\mathcal{A}$ at $f$. Hence $\mathcal{A}$ and $\mathcal{B}$ have the same ramification and are thus Faddeev equivalent.

Now suppose $v \neq 0$. Denote by $c_{u v}$ the value of $f(t)$ at $-u / v$. We prove that $\mathcal{A}$ is Faddeev equivalent to $\mathcal{B}=\left(u+v t, f(t) / c_{u v}\right)$. Indeed, $\mathcal{B}$ can only ramify at $f$, $t+u / v$, and infinity. Since $f(-u / v) / c_{u v}=1, \mathcal{B}$ is unramified at $t+u / v$. It has the same ramification at $f$ as $\mathcal{A}$. Hence $\mathcal{A}$ and $\mathcal{B}$ have the same ramification and are thus Faddeev equivalent.

From Propositions 2.2 and 2.3 we immediately derive

Corollary 2.4. Let $K$ be an arbitrary field, let $F=K(t)$, and let $\mathcal{A}$ be a central simple $F$-algebra of exponent $n$ with at most three (geometric) ramification points such that at least one of them is $K$-rational. Then $\mathcal{A}$ is Faddeev equivalent to a cyclic degree $n$ F-algebra.

As mentioned in the Introduction, this result has an interesting application to cyclicity of $k(x, y)$-algebras ramified along a hyperelliptic curve; see Theorems $\mathrm{A} 5$ and $\mathrm{A} 6$ in the Appendix.

Remark 2.5. Unfortunately we do not know whether the statement of Corollary 2.4 remains true in the case where none of ramification points is $K$-rational ${ }^{2}$ This case may occur (see Proposition 2.6 below). However, in the case where $n$ is prime and $K$ is either local or global, we shall obtain the full result (see Propositions 3.16 and 4.5 where we consider the case $n=2$; the case where $n=p$ is an odd prime can be treated in a similar way).

Proposition 2.6. Let $L$ be a field. Suppose that $L$ has a cyclic extension of degree 3. Let $K=L(z)$ where $z$ is an indeterminate. Then there exists a quaternion algebra over $K(t)$ with ramification only at an irreducible polynomial of degree 3 .

Proof. Let $L(\theta)$ be a cyclic extension of $L$ of degree 3 where $\theta$ is a root of an irreducible polynomial $f \in L[z]$. First we prove that there is an element $a \in K(\theta)$ such that $a \notin\left(K(\theta)^{*}\right)^{2}$ but $N_{K(\theta) / K}(a) \in\left(K^{*}\right)^{2}$. Set $a=(z-\theta) f$. We have $N_{K(\theta) / K}((z-\theta) f)=f^{4} \in\left(K^{*}\right)^{2}$. But $(z-\theta) f \notin\left(K(\theta)^{*}\right)^{2}$ since $(z-\theta) f$ has a root of multiplicity one.

Let $g \in K[t]$ be an irreducible monic polynomial over $K$ such that $(z-\theta) f$ is its root. Then the degree of $g$ is 3 . Consider the quaternion algebra $(-g, t)$ over $K(t)$. This algebra is unramified at infinity since $(-g, t)_{\infty} \sim\left(-t^{3}, t\right) \sim 1$. Since $N_{K(\theta) / K}((z-\theta) f) \in\left(K^{*}\right)^{2}$, we have $-g(0) \in\left(K^{*}\right)^{2}$ and $(-g, t)$ is unramified at $t$. On the other hand, the algebra $(-g, t)$ ramifies at $g$ since $(z-\theta) f \notin\left(K(\theta)^{*}\right)^{2}$. Thus we have constructed a quaternion algebra with ramification only at an irreducible polynomial of degree 3 .

If an algebra is ramified in more than three points, one cannot hope to get a result similar to Corollary 2.4, even in the case of linear ramification. This is clear from the following example (due to D. K. Faddeev and appearing in [16, 3.9] without details).

Proposition 2.7. Let $K$ be an arbitrary field, let $F=K(t)$, and let $\mathcal{A}$ be an algebra over $F$ with ramification $K(\sqrt{a}) / K$ at $t, \infty$ and $K(\sqrt{b}) / K$ at $t-1, t-c$.

${ }^{2}$ Added in proof. We fill this gap in our paper mentioned in footnote 1. 
If $\mathcal{A}$ is Faddeev equivalent to a quaternion algebra, the algebras $((c, b) / K(\sqrt{a}))$ and $((c, a) / K(\sqrt{b}))$ are trivial.

Proof. It is easy to see that the biquaternion algebra

$$
\mathcal{B}=(a, t) \otimes(b,(t-1)(t-c))
$$

has the above invariants.

Assume $((c, b) / K(\sqrt{a})) \nsim 1$. For any algebra $\mathcal{A}$ with the above invariants we have

$$
\mathcal{A} \sim \mathcal{B} \otimes_{F}\left(\mathcal{C} \otimes_{K} F\right)
$$

where $\mathcal{C}$ is some $K$-algebra. Therefore

$$
\mathcal{A}_{t} \sim(a, t) \otimes_{K((t))}(b, c) \otimes_{K((t))}\left(\mathcal{C} \otimes_{K} K((t))\right)
$$

and

$$
\mathcal{A}_{\infty} \sim(a, t) \otimes_{K((1 / t))}\left(\mathcal{C} \otimes_{K} K((1 / t))\right) .
$$

Since $(c, b) \otimes K(\sqrt{a}) \nsim 1$, we conclude that either $(c, b) \otimes \mathcal{C} \otimes K(\sqrt{a}) \nsim 1$ or $\mathcal{C} \otimes K(\sqrt{a}) \nsim 1$

Assume that $(c, b) \otimes \mathcal{C} \otimes K(\sqrt{a}) \nsim 1$. Let $\mathcal{D}$ be a $K$-division algebra similar to $(c, b) \otimes \mathcal{C}$. If $\operatorname{ind}(\mathcal{D}) \geq 4$, then $4 \leq \operatorname{ind}(\mathcal{D} \otimes K((\sqrt{t})))=\operatorname{ind}\left(\mathcal{A}_{t} \otimes K((\sqrt{t}))\right)$. Hence $\operatorname{ind}\left(\mathcal{A}_{t}\right) \geq 4$ and then $\operatorname{ind}(\mathcal{A}) \geq 4$. Thus we may assume $\operatorname{ind}(\mathcal{D})=2=$ $\operatorname{ind}(\mathcal{D} \otimes K(\sqrt{a}))$. But then $(a, t) \otimes \mathcal{D}$ is a division algebra of index 4 , by Corollary 1.14. Hence ind $\left(\mathcal{A}_{t}\right)=4$ and then $\operatorname{ind}(\mathcal{A}) \geq 4$.

In a similar way one can treat the case $\mathcal{C} \otimes K(\sqrt{a}) \nsim 1$.

By an analogous argument the index of either $\mathcal{A}_{\infty}$ or $\mathcal{A}_{t-c}$ is not less than 4 in the case $((c, a) / K(\sqrt{b})) \nsim 1$. Hence the index of $\mathcal{A}$ is not less than 4 .

Let us try to obtain a sufficient condition for an algebra of exponent $n$ to be Faddeev equivalent to a degree $n$ algebra. We restrict ourselves to considering the case $n=2$, where we shall explicitly construct a large family of algebras splitting over a quadratic extension of $F$. This guarantees that the local invariants of every such algebra are good (i.e. the Faddeev index equals 2).

The papers [32] and [33] contain a description of $F$-algebras whose splitting field is isomorphic to the field of rational functions of a hyperelliptic $K$-curve. Such algebras must have good local invariants. Below we describe the invariants of such algebras.

As before, let $K$ be an arbitrary field, let $F=K(t)$, and let $\mathcal{A}$ be a central simple $F$-algebra of exponent 2 . Before describing the systems of local invariants of algebras split by the function field of a split hyperelliptic curve, let us first introduce some additional notation.

Let $m$ be odd, and let $C$ denote the (split) hyperelliptic curve over $K$ corresponding to the affine curve

$$
y^{2}=\left(t-a_{1}\right) \ldots\left(t-a_{m}\right) .
$$

Suppose $c_{1}, \ldots, c_{r} \in \bar{K}$, the separable closure of $K$, satisfy

$$
\left(c_{j}-a_{1}\right) \ldots\left(c_{j}-a_{m}\right) \in\left(K\left(c_{j}\right)^{*}\right)^{2}, j=1, \ldots, r .
$$

Denote

$$
F^{\prime}=F\left(\sqrt{\left(t-a_{1}\right) \ldots\left(t-a_{m}\right)}\right)=K(C) .
$$


Let $N_{L / K}: L^{*} \rightarrow K^{*}$ denote the norm map $\left(L^{*}, K^{*}\right.$ denote the multiplicative groups of $L, K$, respectively).

It follows from 32 that the algebras

$$
\mathcal{A}_{c_{1}, \ldots, c_{r}}=\bigotimes_{i=1}^{m-1}\left(\prod_{j=1}^{r} N_{K\left(c_{j}\right) / K}\left(c_{j}-a_{i}\right),\left(t-a_{i}\right)\left(t-a_{m}\right)\right)
$$

and

$$
\mathcal{B}_{s}=\bigotimes_{i=1, i \neq s}^{m-1}\left(a_{s}-a_{i},\left(t-a_{i}\right)\left(t-a_{m}\right)\right) \otimes\left(\prod_{j=1, j \neq s}^{m}\left(a_{j}-a_{s}\right),\left(t-a_{s}\right)\left(t-a_{m}\right)\right)
$$

split over $F^{\prime}$ and therefore have index at most 2. See [27] for a more detailed discussion.

Let

$$
u_{i}=\prod_{j=1}^{r} N_{K\left(c_{j}\right) / K}\left(c_{j}-a_{i}\right), i=1, \ldots, m-1,
$$

$u_{m}=\prod_{i=1}^{m-1} u_{i}$. For $1 \leq s \leq m$ set $w_{s, i}=a_{s}-a_{i}, i \neq m, s, w_{s, i}=\prod_{j=1, j \neq i}^{m}\left(a_{j}-a_{i}\right)$ for $i=m, s$.

Note that $\mathcal{A}_{c_{1}, \ldots, c_{r}}$ and $\mathcal{B}_{s}$ at finite points have local invariants $\left\{t-a_{i}, K\left(\sqrt{u_{i}}\right) / K\right\}$ and $\left\{t-a_{i}, K\left(\sqrt{w_{s, i}}\right) / K\right\}$, respectively.

Denote by $M$ the power set $\mathcal{P}(\{1, \ldots, m\})$. For $1 \leq j \leq m$ and $M^{\prime} \in M$ let

$$
W_{j, M^{\prime}}=\prod_{s \in M^{\prime}} w_{s, j}
$$

If $M^{\prime}=\varnothing$, we set $W_{j, M^{\prime}}=1$.

Proposition 2.8. Suppose that $m>1$ is odd. Let $\mathcal{A}$ be an $F$-algebra with local invariants at finite points

$$
\left\{t-a_{i}, K\left(\sqrt{u_{i} W_{i, M^{\prime}}}\right) / K\right\}
$$

for some $M^{\prime} \in M$. Then $\mathcal{A}$ is Faddeev equivalent to a quaternion algebra.

Proof. Indeed, such an $\mathcal{A}$ is Faddeev equivalent to the algebra $\bigotimes_{s \in M^{\prime}} \mathcal{B}_{s} \otimes \mathcal{A}_{c_{1}, \ldots, c_{r}}$. Since the latter algebra splits over a quadratic extension, $\mathcal{A}$ is Faddeev equivalent to a quaternion algebra.

Now let $m>2$ be even, and let $C$ be the hyperelliptic curve corresponding to the affine curve

$$
y^{2}=\left(t-b_{2}\right) \ldots\left(t-b_{m}\right),
$$

where $b_{i}=-\prod_{j=2, j \neq i}^{m}\left(a_{j}-a_{1}\right)$. Similar to the case of odd $m$ we can construct algebras of index at most 2. Suppose $c_{1}, \ldots, c_{r} \in \bar{K}$ satisfy

$$
\left(c_{j}-b_{2}\right) \ldots\left(c_{j}-b_{m}\right) \in\left(K\left(c_{j}\right)^{*}\right)^{2}, j=1, \ldots, r .
$$

We proceed as follows (see [33). Denote

$$
z_{i}=\left(a_{m}-a_{1}\right)\left(a_{i}-a_{1}\right)\left(t-a_{i}\right)\left(t-a_{m}\right), \quad i=2, \ldots, m-1 .
$$

Then the algebras

$$
\mathcal{C}_{c_{1}, \ldots, c_{r}}=\bigotimes_{i=2}^{m-1}\left(\prod_{j=1}^{r}\left(N_{K\left(c_{j}\right) / K}\left(c_{j}+\prod_{l=2, l \neq i}^{m}\left(a_{l}-a_{1}\right)\right)\right), z_{i}\right)
$$


and

$$
\begin{gathered}
\mathcal{D}_{s}=\bigotimes_{i=2, i \neq s}^{m-1}\left(-\prod_{l=2, l \neq s}^{m}\left(a_{l}-a_{1}\right)+\prod_{l=2, l \neq i}^{m}\left(a_{l}-a_{1}\right), z_{i}\right) \\
\otimes\left(\prod_{j=2, j \neq s}^{m}\left(-\prod_{l=2, l \neq j}^{m}\left(a_{l}-a_{1}\right)+\prod_{l=2, l \neq s}^{m}\left(a_{l}-a_{1}\right)\right), z_{s}\right)
\end{gathered}
$$

have indices at most 2 since all of them split over $F^{\prime}$ (defined in (2.2)).

Also let

$$
u_{i}=\prod_{j=1}^{r}\left(N_{K\left(c_{j}\right) / K}\left(c_{j}+\prod_{l=2, l \neq i}^{m}\left(a_{l}-a_{1}\right)\right)\right), i=2, \ldots, m-1,
$$

$u_{1}=1, u_{m}=\prod_{i=2}^{m} u_{i}$. Let $1<s<m$ and

$$
\begin{aligned}
& w_{s, i}=-\prod_{l=2, l \neq s}^{m}\left(a_{l}-a_{1}\right)+\prod_{l=2, l \neq i}^{m}\left(a_{l}-a_{1}\right), i \neq 1, s, \\
& w_{s, s}=\prod_{j=2, j \neq s}^{m}\left(-\prod_{l=2, l \neq j}^{m}\left(a_{l}-a_{1}\right)+\prod_{l=2, l \neq s}^{m}\left(a_{l}-a_{1}\right)\right),
\end{aligned}
$$

$w_{s, m}=\prod_{i=2}^{m-1} w_{s, i}, w_{s, 1}=1$.

Let $M=\mathcal{P}(\{1, \ldots, m\})$ and

$$
W_{j, M^{\prime}}=\prod_{s \in M^{\prime}} w_{s, j}
$$

for $1 \leq j \leq m$ and $M^{\prime} \in M$.

Proposition 2.9. Suppose that $m>1$ is even. Let $\mathcal{A}$ be an F-algebra with local invariants at finite points

$$
\left\{t-a_{i}, K\left(\sqrt{u_{i} W_{j, M^{\prime}}}\right) / K\right\}
$$

for some $M^{\prime} \in M$. Then $\mathcal{A}$ is Faddeev equivalent to a quaternion algebra.

Proof. As before, any such $\mathcal{A}$ is Faddeev equivalent to the algebra $\bigotimes_{s \in M^{\prime}} \mathcal{D}_{s} \otimes$ $\mathcal{C}_{c_{1}, \ldots, c_{r}}$ which splits over a quadratic extension.

\section{LOCAL FIELD CASE}

In this section we consider the case where $K$ is an $\ell$-adic field. (We always restrict our attention to algebras of prime exponent $p$ different from $\ell$.)

Our main goal is to produce examples of "bad" systems of invariants, or, equivalently, algebras of Faddeev index $>p$ (see Theorem 3.6). In the special case where an algebra is ramified at three points we show that its invariants are always "good" (Proposition 3.16). In the case of linear ramification we are fully able to distinguish between "good" and "bad" invariants (see Proposition 3.10).

First we describe all central simple algebras of exponent $p$ over $F=K(t)$ in terms of the Faddeev theory. Let $\beta \in{ }_{p} \operatorname{Br}(F)$. By Bloch's theorem, $\beta$ is a product of symbols. Since one can multiply by $p$-powers without affecting symbols, we may assume the symbols are in $K[t]$, and thus of the form $(a f, b g)$, where $f, g \in K[t]$ are monic and $a, b \in K$. Furthermore, we could decompose the algebra further unless $f, g$ are irreducible, so we make this further assumption. 
To calculate the ramification of a symbol $(f, g)$ at $\infty$, where $f, g \in K[t]$, we suppose that the leading monomials of $f, g$ are respectively $a t^{m}, b t^{n}$. Recall that the residue of $(f, g)$ at any discrete valuation $v$ (including the valuation corresponding to the infinite point) is defined by the $p$ th root of the residue of $c=$ $(-1)^{v(f) v(g)} f^{v(g)} / g^{v(f)}$. At $v=\infty$ we have $c=(-1)^{m n} a^{-n} b^{m}$.

Recall that every element of $K \bmod p$-powers has the form $\alpha^{i} \pi^{j}$, where $\pi$ is a (fixed) uniformizing parameter of $K$, and $\alpha$ has non-p-power residue in $k$. Now note that $\left(a^{i}, f\right) \sim\left(a, f^{i}\right)$. In particular, since

$$
(a f, b g) \sim(a, b) \otimes(a, g) \otimes(f, b) \otimes(f, g),
$$

we can take $a, b \in\{1, \alpha, \pi, \alpha \pi\}$, and thus each symbol is Faddeev equivalent to a product of symbols of the form:

TYPE I $(\alpha, f)$;

TYPE II $(\pi, g)$;

TYPE III $\left(h_{1}, h_{2}\right)$.

Here $f, g, h_{1}, h_{2}$ are assumed monic irreducible (if $p$ is odd); for $p=2$ we assume instead that $(-1)^{\operatorname{deg} h_{2}} h_{2}$ is monic. This assumption implies that any algebra of type III is unramified at infinity. An algebra of type I is unramified at $\infty$ if $\operatorname{deg} f$ is a multiple of $p$ and has ramification $K\left(\sqrt[p]{\alpha^{m}}\right) / K$ for some $m$ if $\operatorname{deg} f$ is not a multiple of $p$. Likewise an algebra of type II is unramified at $\infty$ if $\operatorname{deg} g$ is a multiple of $p$ and has ramification $K\left(\sqrt[p]{\pi^{n}}\right) / K$ if $\operatorname{deg} g$ is not a multiple of $p$. Thus $\mathcal{A}$ is unramified at $\infty$ unless $f$ or $g$ has degree not a multiple of $p$.

Of course the product of symbols of type I has type I, and likewise for type II. In conclusion we have

Proposition 3.1. Let $\mathcal{A}$ be any central simple algebra over $K(t)$ of prime exponent p. Then $\mathcal{A}$ is Faddeev equivalent to a tensor product

$$
(\alpha, f) \otimes(\pi, g) \otimes\left(\bigotimes\left(h_{1 i}, h_{2 i}\right)\right) .
$$

Algebras with linear ramification admit a more precise description than the general one in Proposition 3.1 because we can eliminate type III above.

Proposition 3.2. If $\mathcal{A}$ has linear ramification, then it is Faddeev equivalent to an algebra of the form $(f, \alpha) \otimes(g, \pi)$, where $f, g$ are split monic polynomials.

Proof. If $\mathcal{A}$ is ramified at linear polynomials $t-a_{i}$ and $K\left(\sqrt[p]{b_{i}}\right) / K$ is the ramification at $t-a_{i}$, then the algebra $\bigotimes_{i}\left(b_{i}, t-a_{i}\right)$ has the same invariants. Since $p$ is prime to the residue characteristic of $K$, we may (and will) assume that $b_{i} \in\left\{\alpha, \pi, \alpha \pi, \ldots, \alpha^{p-1} \pi\right\}$. It only remains to decompose each symbol containing $\alpha \pi$ into a product of two symbols and then collect all symbols containing $\alpha$ as well as those containing $\pi$.

Our next goal is to produce a large family of algebras of exponent $p$ whose local invariants are bad, i.e. algebras not Faddeev equivalent to a cyclic algebra. We mainly deal with the case $p=2$ and postpone the case of odd $p$ until Lemma 3.8.

The first known example of a division algebra of index 4 and exponent 2 over $K(t)$ is the Jacob-Tignol example published in [29, Appendix]. The fact that it is a division algebra is proved using a result which motivates Example 3.7 below.

Example 3.3 (Jacob-Tignol). Let $K$ be a nondyadic local field, and let $\alpha$ be a unit nonsquare as above. Suppose $g \in K[t]$ has constant term 1 , and the residue of 
$g$ (with respect to $\pi$ ) is a nonsquare. (Their concrete example was $g=t+1$.) Then the biquaternion algebra $\mathcal{A}=(t, \alpha) \otimes(g, \pi)$ over $F$ is actually a division algebra (where $\alpha \in K$ is not a square modulo $\pi$ ).

Remark 3.4. Note that the algebra $\mathcal{A}$ from the Jacob-Tignol example has good local invariants since

$$
\begin{aligned}
(-\pi t, \alpha(t+1)) & \sim(\pi, \alpha(t+1)) \otimes(-t, \alpha) \otimes(-t, t+1) \\
& \sim(\pi, \alpha(t+1)) \otimes(-t, \alpha) \sim \mathcal{A} \otimes(\alpha, \pi)
\end{aligned}
$$

because $-t+(t+1)=1$ and $(-1, \alpha)$ is trivial.

So let us search for bad systems of local invariants.

Let $\mathcal{A}=(f, \alpha) \otimes(g, \pi)$, where $\alpha \in R^{*}$ is nonsquare and $f, g \in R[t]$. We want to find symbols which do not reduce the index of $\mathcal{A}$.

Lemma 3.5. Suppose $\mathcal{A}=(f, \alpha) \otimes(g, \pi)$, where $f, g \in R[t]$, the reduction $\bar{g}$ is not a square, $\bar{f}$ has a root of odd multiplicity $\bar{a} \in k$, and $\bar{g}(\bar{a}) \bar{\alpha}$ is not a square. Then $\mathcal{A}$ has index 4.

Proof. If the assertion is false, the Albert form $\langle f, \alpha,-\alpha f,-g,-\pi, \pi g\rangle$ is isotropic over $F$. By Springer's theorem (Theorem 1.11) applied to residues modulo $\pi$, either $\langle-1, \bar{g}\rangle$ or $\langle\bar{f}, \bar{\alpha},-\bar{\alpha} \bar{f},-\bar{g}\rangle$ is isotropic over $k(t)$. Since by hypothesis the first form is anisotropic, the second one must be isotropic. We write

$$
\bar{f}=(t-\bar{a})^{m} \bar{h}
$$

with $m$ odd, $\bar{h}(\bar{a}) \neq 0$, and apply Springer's theorem once again, this time with respect to the valuation on $k(t)$ defined by $t-\bar{a}$. We conclude that either $\langle-\bar{\alpha}, \bar{g}(\bar{a})\rangle$ or $\langle\bar{h}(\bar{a}),-\bar{\alpha} \bar{h}(\bar{a})\rangle=\langle\bar{h}(\bar{a})\rangle \cdot\langle 1,-\bar{\alpha}\rangle$ is isotropic over $k$, which contradicts the hypothesis.

Theorem 3.6. Suppose in the notation of Lemma 3.5 that $\bar{f}$ has two roots $\bar{a}$ and $\bar{b}$, both of odd multiplicity, such that $\bar{g}(\bar{a}) \bar{\alpha}$ and $\bar{g}(\bar{b})$ are not squares in $k$. Then $\mathcal{A}$ and $\mathcal{A} \otimes(\pi, \alpha)$ both have index 4 , and thus $\mathcal{A}$ has bad local invariants.

Proof. $\mathcal{A}$ is a division algebra by Lemma 3.5, and $\mathcal{A} \otimes(\pi, \alpha) \sim(f, \alpha) \otimes(g \alpha, \pi)$, so apply Lemma 3.5 using $b$ instead of $a$, and $g \alpha$ instead of $g$.

Example 3.7. Take $f=t(t-1)$. To apply Theorem 3.6 we need $\bar{g}(0) \bar{\alpha}$ and $\bar{g}(1)$ (or $g(1) \bar{\alpha}$ and $\bar{g}(0))$ not to be squares.

(i) If $g$ is linear, then $g=t+c$, so $\overline{c \alpha}$ and $\overline{c+1}$ must be nonsquares. The former implies $\bar{c}=\overline{d^{2}}$, and thus $\overline{d^{2}+1}$ must be nonsquare. This is well known to have a solution, since any element in a finite field is a sum of two squares.

(ii) More generally the same argument works if $g=t^{m}+c$ for $m$ arbitrary. For $m$ even there is no ramification at infinity.

Our next results generalize Theorem 3.6 in the case of linear ramification.

Note that every $K$-division algebra of degree $p$ is Brauer equivalent to $\left(\pi, \alpha^{j}\right)$ (because it can be written as $(\pi, \alpha)^{\otimes j}$ ). We need the following

Lemma 3.8. $(\alpha, f) \otimes\left(\pi, \alpha^{i} g\right)$ has Faddeev index $p^{2}$ unless $K\left(\sqrt[p]{\alpha^{j} g}\right)$ splits $(\alpha, f)$ for some $j$.

Proof. Otherwise we have $(\alpha, f) \otimes\left(\pi, \alpha^{i} g\right) \sim\left(\pi, \alpha^{j}\right) \otimes \mathcal{D}$ for $\mathcal{D}$ a division algebra of degree $\leq p$, so $\mathcal{D} \sim(\alpha, f) \otimes\left(\pi, \alpha^{i-j} g\right)$. We conclude by Proposition 1.13 unless $K\left(\sqrt[p]{\alpha^{i-j} g}\right)$ splits $(\alpha, f)$. 
Let $\mathcal{A}$ be a central simple $F$-algebra of exponent $p$. Suppose that $\mathcal{A}$ has ramification at $n$ linear points $t-a_{l}, l=1, \ldots, n$. Then for suitable squarefree products $f, g$ of the $t-a_{l}$, the algebra $\mathcal{A}$ is Faddeev equivalent to all tensor products

$$
\mathcal{B}_{i}=(\alpha, f)_{p} \otimes\left(\pi, \alpha^{i} g\right)_{p}
$$

where $0 \leq i \leq p-1$ (and hence Brauer equivalent to one of them).

Proposition 3.9. Let $\bar{a}_{l} \neq \bar{a}_{j}, l \neq j$. In the above notation, if $\mathcal{A}$ is Faddeev equivalent to an algebra of index $p$, then $f$ has no roots $b_{0}, \ldots, b_{p-1}$ such that $\alpha^{i} g\left(b_{i}\right) \in\left(K^{*}\right)^{p}, 0 \leq i \leq p-1$.

Proof. If for any $i$ there is a root $b_{i}$ of $f$ such that $\alpha^{i} g\left(b_{i}\right) \in\left(K^{*}\right)^{p}$, then the algebras

$$
\overline{\mathcal{B}_{i}}=(\bar{\alpha}, \bar{f})_{p} \otimes k(t)\left(\sqrt[p]{\overline{\alpha^{i} g}}\right)
$$

are ramified at the points $t-\overline{b_{i}}$. Hence all $\overline{\mathcal{B}_{i}}$ 's are division algebras. Therefore by Corollary 1.14 the algebras $\mathcal{B}_{i}, i=0, \ldots, p-1$, have index $p^{2}$, and, by Lemma 3.8, $\mathcal{A}$ cannot be Faddeev equivalent to an algebra of index $p$.

In the case of algebras of exponent 2 we shall strengthen the previous result formulating a criterion for such an algebra to be Faddeev equivalent to a quaternion algebra.

Let $\mathcal{A}$ be a central simple $F$-algebra of exponent 2. Suppose that $\mathcal{A}$ has ramification at $m$ linear points $t-a_{i}, i=1, \ldots, m$. Then by Proposition 3.2 , $\mathcal{A}$ is Faddeev equivalent to a tensor product

$$
\mathcal{B}=(\alpha, f)_{2} \otimes(\pi, g)_{2},
$$

where $f, g$ are products of some of the $t-a_{i}$ with no repetition. We assume that $g \neq 1$ (if $g=1, \mathcal{A}$ is obviously a quaternion algebra) and $\bar{a}_{i} \neq \bar{a}_{j}$ for $i \neq j$.

First note that without loss of generality one can assume that $\operatorname{deg} g$ is odd. Indeed, since $g \neq 1$, then some linear point has ramification $K(\sqrt{\pi}) / K$ or $K(\sqrt{\alpha \pi}) / K$. Using the automorphism group of $\mathbb{P}_{K}^{1}$, one may assume that $\mathcal{A}$ is ramified at infinity with ramification $K(\sqrt{\pi}) / K$ or $K(\sqrt{\alpha \pi}) / K$. In such a case the degree of the "new" $g$ is odd.

Proposition 3.10. With the above notation, suppose $\bar{a}_{i} \neq \bar{a}_{j}, i \neq j$. Assume that $\operatorname{deg} g$ is odd. Then $\mathcal{A}$ is Faddeev equivalent to a quaternion algebra if and only if there are no roots $b_{1}, b_{2}$ of $f$ such that $g\left(b_{1}\right), \alpha g\left(b_{2}\right) \notin K^{2}$.

We start by proving

Lemma 3.11. Let $C$ be a hyperelliptic curve over $K$ with good reduction, and let $\mathcal{D}$ be a central simple unramified algebra over the function field $K(C)$ such that $\mathcal{D} \nsim 1$ and $\mathcal{D} \nsim(\alpha, \pi) \otimes K(C)$. Then $\mathcal{D} \otimes K(\sqrt{\alpha})(C) \nsim 1$.

Proof. In [36] the 2-torsion elements of the Brauer group of a hyperelliptic curve $C$ with good reduction are represented by unramified algebras. In [36] it is also shown that the algebras representing nonconstant elements remain nontrivial over $L(C)$, where $L$ is an unramified extension of $K$. The lemma is proved.

Proof of Proposition 3.10. If such roots exist, then $\bar{g}\left(\bar{b}_{1}\right), \overline{\alpha g}\left(\bar{b}_{2}\right) \notin\left(k^{*}\right)^{2}$, and $\mathcal{A}$ is not Faddeev equivalent to a quaternion algebra, by Theorem [3.6. To prove the converse, we must consider four possibilities:

1) all roots of $f$ are also roots of $g$; 
2) only one root $b$ of $f$ is not a root of $g$;

3) for any root $b$ of $f$ such that $g(b) \neq 0$, one has $g(b) \in\left(K^{*}\right)^{2}$;

4) for any root $b$ of $f$ such that $g(b) \neq 0$, one has $\alpha g(b) \in\left(K^{*}\right)^{2}$.

In case 1) we conclude that the algebra $\mathcal{B} \otimes F(\sqrt{g}) \sim(\alpha, f) \otimes F(\sqrt{g})$ is unramified. (The fact that it is unramified at infinity follows from the assumption on the degree of $g$.) Since the curve defined by the equation $y^{2}=g(t)$ has good reduction, from Lemma 3.11 it follows that $(\alpha, f) \otimes F(\sqrt{g})$ is trivial or $(\alpha, f) \otimes F(\sqrt{g}) \sim$ $(\alpha, \pi) \otimes F(\sqrt{g})$. In the first case the algebra $\mathcal{B}$ has index 2 . In the second case the algebra $\mathcal{B} \otimes(\alpha, \pi)$ has index 2 .

In case 2) the algebra $(\alpha, f) \otimes(\pi, \alpha g(b) g) \otimes F(\sqrt{\alpha g(b) g}) \sim(\alpha, f) \otimes F(\sqrt{\alpha g(b) g})$ is unramified. Indeed, it is unramified outside the point defined by the polynomial $t-b$. Note that the completion of $F(\sqrt{\alpha g(b) g})$ with respect to the valuation extending the valuation of $F$ corresponding to $t-b$ is

$$
F(\sqrt{\alpha g(b) g(b)})((t-b))=F(\sqrt{\alpha})((t-b)) .
$$

Then

$$
(\alpha, f)_{t-b}=(\alpha, f) \otimes F(\sqrt{\alpha})((t-b)) \sim 1 .
$$

Thus $(\alpha, f) \otimes F(\sqrt{\alpha g(b) g})$ is unramified. Hence as in case 1$)$ the algebra $\mathcal{B}$ is Faddeev equivalent to a quaternion algebra.

As to case 3), the algebra $(\alpha, f) \otimes(\pi, \alpha g) \otimes F(\sqrt{\alpha g}) \sim(\alpha, f) \otimes F(\sqrt{\alpha g})$ is unramified outside the points defined by the polynomials $t-a_{i}$, where $f\left(a_{i}\right)=0$, $g\left(a_{i}\right) \neq 0$. The completion of $F(\sqrt{\alpha g})$ with respect to the valuation extending the valuation of $F$ corresponding to $t-a_{i}$ is

$$
F\left(\sqrt{\alpha g\left(a_{i}\right)}\right)\left(\left(t-a_{i}\right)\right)=F(\sqrt{\alpha})\left(\left(t-a_{i}\right)\right) .
$$

Then the algebra $(\alpha, f) \otimes F(\sqrt{\alpha g})$ is unramified. From Lemma 3.11 it follows that $(\alpha, f) \otimes F(\sqrt{\alpha g})$ is trivial or $(\alpha, f) \otimes F(\sqrt{\alpha g}) \sim(\alpha, \pi) \otimes F(\sqrt{\alpha g})$. Therefore the algebra $\mathcal{B}$ is Faddeev equivalent to a quaternion algebra.

Likewise, in case 4) the algebra $(\alpha, f) \otimes(\pi, g) \otimes F(\sqrt{g})$ is unramified. Hence the algebra $\mathcal{B}$ is Faddeev equivalent to a quaternion algebra.

Thus in cases 1)-4) we get algebras Faddeev equivalent to quaternion algebras. The proposition is proved.

Our next results show that one can significantly enlarge the supply of algebras with bad local invariants if one does not restrict oneself to considering only tensor products of algebras of certain types. Namely, in the next proposition we present an example showing that not every algebra is equivalent to an algebra of the form $(f, \alpha) \otimes(g, \pi)$.

Proposition 3.12. Let $\mathcal{A}=\left(\pi, t^{2}-\alpha+\alpha^{2}\right)_{2} \otimes_{F}\left(t, t^{2}-\alpha\right)_{2}$. Then $\mathcal{A} \otimes_{F} F(\sqrt{\alpha})$ has index 4.

Proof. We have

$$
\begin{aligned}
\mathcal{A} \otimes_{F} F(\sqrt{\alpha}) & =\left(\pi, t^{2} / \alpha-1+\alpha\right) \otimes\left(t, t^{2} / \alpha-1\right) \\
& \sim\left(\pi,(t / \sqrt{\alpha})^{2}-1+\alpha\right) \otimes\left(t,(t / \sqrt{\alpha})^{2}-1\right) .
\end{aligned}
$$

Set $z=\frac{t}{\sqrt{\alpha}}$. The algebra $\mathcal{A} \otimes_{F} F(\sqrt{\alpha})$ has index 4 if and only if

$$
\left(\pi, z^{2}-1+\alpha\right) \otimes\left(\sqrt{\alpha} z, z^{2}-1\right)
$$

is also of index 4 . 
The latter algebra has index 4 if and only if the Albert form

$$
\left\langle\pi, z^{2}-1+\alpha,-\pi\left(z^{2}-1+\alpha\right),-\sqrt{\alpha} z,-\left(z^{2}-1\right), \sqrt{\alpha} z\left(z^{2}-1\right)\right\rangle
$$

is anisotropic over $K(\sqrt{\alpha})(z)$.

By Springer's theorem applied to the residues modulo $\pi$, we conclude that this form is anisotropic if the forms

$$
\left\langle 1,-\left(z^{2}-1+\bar{\alpha}\right)\right\rangle \quad \text { and } \quad\left\langle z^{2}-1+\bar{\alpha},-\sqrt{\bar{\alpha}} z,-\left(z^{2}-1\right), \sqrt{\bar{\alpha}} z\left(z^{2}-1\right)\right\rangle
$$

are anisotropic over the field $k(\sqrt{\bar{\alpha}})(z)$. The first form is anisotropic since $z^{2}-1+\bar{\alpha}$ is not a square in $k(\sqrt{\bar{\alpha}})(z)$. Let us thus consider the form

$$
\left\langle z^{2}-1+\bar{\alpha},-\sqrt{\bar{\alpha}} z,-\left(z^{2}-1\right), \sqrt{\bar{\alpha}} z\left(z^{2}-1\right)\right\rangle .
$$

By Springer's theorem applied to the valuation defined by $z-1$, it is anisotropic over $k(\sqrt{\bar{\alpha}})(z)$ if the forms $\langle\bar{\alpha},-\sqrt{\bar{\alpha}}\rangle$ and $\langle-1, \sqrt{\bar{\alpha}}\rangle$ are anisotropic over $k(\sqrt{\bar{\alpha}})$. This is obviously the case. The proposition is proved.

Proposition 3.12 also shows that there are algebras which do not admit a representation in the form $(\alpha, f) \otimes\left(h_{1}, h_{2}\right)$. It turns out that there are also algebras which do not admit a representation in the form $(\pi, g) \otimes\left(h_{1}, h_{2}\right)$ (even if we are allowed to change the uniformizer).

Proposition 3.13. Suppose

$$
\mathcal{A}=\left(t,\left(t^{p}-\pi\right) \ldots\left(t^{p}-\alpha^{p-1} \pi\right)\right)_{p} \otimes_{F}\left(\alpha,\left(t^{p}-a^{p^{2}} \pi\right) \ldots\left(t^{p}-\alpha^{p-1} a^{p^{2}} \pi\right)\right)_{p},
$$

where $a$ is a unit such that

$$
\left(a^{p^{2}}-\alpha^{-s}\right) \ldots\left(a^{p^{2}}-\alpha^{p-1-s}\right) \in\left(K^{*}\right)^{p}, s=0, \ldots, p-1 .
$$

Then the algebras

$$
\mathcal{A} \otimes_{F} K\left(\sqrt[p]{\alpha^{s} \pi}\right)(t), s=0, \ldots, p-1
$$

have index $p^{2}$.

Proof. Consider the algebra $\mathcal{A} \otimes_{F} K\left(\sqrt[p]{\alpha^{s} \pi}\right)(t)$. Set $\alpha^{s} \pi=\pi^{\prime}, \Pi=\sqrt[p]{\pi^{\prime}}$. Then

$$
\begin{aligned}
\mathcal{A} & \otimes_{F} K(\Pi)(t) \sim\left(t,\left(t^{p}-\pi^{\prime} \alpha^{-s}\right) \ldots\left(t^{p}-\alpha^{p-1-s} \pi^{\prime}\right)\right) \\
& \otimes\left(\alpha,\left(t^{p}-a^{p^{2}} \alpha^{-s} \pi^{\prime}\right) \ldots\left(t^{p}-\alpha^{p-1} a^{p^{2}} \alpha^{p-1-s} \pi^{\prime}\right)\right) \otimes_{F} K(\Pi)(t) .
\end{aligned}
$$

We have

$$
\begin{aligned}
\mathcal{A} & \otimes_{K(t)} K(\Pi)(t)=\left(t,\left(t^{p}-\Pi^{p} \alpha^{-s}\right) \ldots\left(t^{p}-\alpha^{p-1-s} \Pi^{p}\right)\right) \\
& \otimes\left(\alpha,\left(t^{p}-a^{p^{2}} \alpha^{-s} \Pi^{p}\right) \ldots\left(t^{p}-a^{p^{2}} \alpha^{p-1-s} \Pi^{p}\right)\right) \otimes K(\Pi)(t) .
\end{aligned}
$$

Set $z=\frac{t}{\Pi}$. Then we obtain an algebra

$$
\begin{aligned}
\mathcal{B}= & \left(\Pi z,\left(z^{p}-\alpha^{-s}\right) \ldots\left(z^{p}-\alpha^{p-1-s}\right)\right) \\
& \otimes\left(\alpha,\left(z^{p}-a^{p^{2}} \alpha^{-s}\right) \ldots\left(z^{p}-a^{p^{2}} \alpha^{p-1-s}\right)\right) .
\end{aligned}
$$

Consider the algebra $\mathcal{B} \otimes K(\Pi)(z)(\sqrt[p]{z})$. Let $w^{p}=z$. Then

$$
\begin{aligned}
\mathcal{B} \otimes K(\Pi)(z)(\sqrt[p]{z}) & =\left(\Pi,\left(w^{p^{2}}-\alpha^{-s}\right) \ldots\left(w^{p^{2}}-\alpha^{p-1-s}\right)\right) \\
& \otimes\left(\alpha,\left(w^{p^{2}}-a^{p^{2}} \alpha^{-s}\right) \ldots\left(w^{p^{2}}-a^{p^{2}} \alpha^{p-1-s}\right)\right) .
\end{aligned}
$$

Let

$$
\begin{aligned}
\overline{\mathcal{B}} & =\left(\bar{\alpha},\left(w^{p^{2}}-\bar{a}^{p^{2}} \bar{\alpha}^{-s}\right) \ldots\left(w^{p^{2}}-\bar{a}^{p^{2}} \bar{\alpha}^{p-1-s}\right)\right) \\
& \otimes k(w)\left(\sqrt[p]{\left(w^{p^{2}}-\bar{\alpha}^{-s}\right) \ldots\left(w^{p^{2}}-\bar{\alpha}^{p-1-s}\right)}\right) .
\end{aligned}
$$


As in Corollary 1.14 we conclude that $\mathcal{B} \otimes K(\Pi)(z)(\sqrt[p]{z})$ has index $p^{2}$ if $\overline{\mathcal{B}}$ is a division algebra.

The completion of $k(w)\left(\sqrt[p]{\left(w^{p^{2}}-\bar{\alpha}^{-s}\right) \ldots\left(w^{p^{2}}-\bar{\alpha}^{p-1-s}\right)}\right)$ with respect to the valuation extending the valuation $v_{w-\bar{a}}$ of $k(w)$ is

$$
k\left(\sqrt[p]{\left(\bar{a}^{p^{2}}-\bar{\alpha}^{-s}\right) \ldots\left(\bar{a}^{p^{2}}-\bar{\alpha}^{p-1-s}\right)}\right)((w-\bar{a}))=k((w-\bar{a})) .
$$

Note that $w-\bar{a}$ is a divisor of multiplicity one of the polynomial $\left(w^{p^{2}}-\bar{a}^{p^{2}} \bar{\alpha}^{-s}\right)$ $\ldots\left(w^{p^{2}}-\bar{a}^{p^{2}} \bar{\alpha}^{p-1-s}\right)$. Let $\left(w^{p^{2}}-\bar{a}^{p^{2}} \bar{\alpha}^{-s}\right) \ldots\left(w^{p^{2}}-\bar{a}^{p^{2}} \bar{\alpha}^{p-1-s}\right)=(w-\bar{a}) h(w)$. Since $\operatorname{Br}(k)$ is trivial, the algebra

$$
\begin{gathered}
\overline{\mathcal{B}}_{w-\bar{a}}=\mathcal{B} \otimes k((w-\bar{a})) \\
\sim(\bar{\alpha}, w-\bar{a}) \otimes(\bar{\alpha}, h(\bar{a})) \otimes k((w-\bar{a})) \sim(\bar{\alpha}, w-\bar{a}) \otimes k((w-\bar{a}))
\end{gathered}
$$

is ramified. Hence it is nontrivial. Then $\overline{\mathcal{B}}$ is a division algebra, and $\mathcal{B}$ has index $p^{2}$. Thus if $\left(a^{p^{2}}-\alpha^{-s}\right) \ldots\left(a^{p^{2}}-\alpha^{p-1-s}\right) \in\left(K^{*}\right)^{p}$ for $s=0, \ldots, p-1$, then all algebras (3.2) have index $p^{2}$. The proposition is proved.

To prove the existence of fields with condition (3.1), consider the following system of equations over $k$ :

$$
\left(x^{p^{2}}-\bar{\alpha}^{-s}\right) \ldots\left(x^{p^{2}}-\bar{\alpha}^{p-1-s}\right)=y_{s}^{p}, s=0, \ldots, p-1 .
$$

Let $\rho$ be a primitive $p$ th root of 1 . If $\rho \in K^{*} \backslash\left(K^{*}\right)^{p}$, one can set $\alpha=\rho$. The sets $\left\{\bar{\rho}^{-s}, \ldots, \bar{\rho}^{p-1-s}\right\}, s=0, \ldots, p-1$, contain all the $p$ th roots of unity and therefore coincide. Thus this system has a solution if and only if the equation

$$
\left(x^{p^{2}}-1\right)\left(x^{p^{2}}-\bar{\rho}\right) \ldots\left(x^{p^{2}}-\bar{\rho}^{p-1}\right)=y^{p}
$$

has a solution. Since this equation defines an absolutely irreducible $k$-variety, we can apply [24, Th. 1] to conclude that this equation has a solution in some extension of $k$ of degree prime to $p$. For a field $K$ with such residue field condition, (3.1) is satisfied.

Now we will show that there are infinitely many $\ell$-adic fields $K$ with the condition $\rho \in K^{*} \backslash\left(K^{*}\right)^{p}$. Denote by $\rho_{n}$ an $n$th primitive root of unity, e.g., $\rho_{p}=\rho$. Then $\left[\mathbb{Q}\left(\rho_{p^{2}}\right): \mathbb{Q}\left(\rho_{p}\right)\right]=p,\left[\mathbb{Q}\left(\rho_{p}\right): \mathbb{Q}\right]=p-1$, and for any prime $\ell \neq p$ the extension $\mathbb{Q}_{\ell}\left(\rho_{p^{2}}\right) / \mathbb{Q}_{\ell}\left(\rho_{p}\right)$ is Galois of degree dividing $p$. If $\left[\mathbb{Q}_{\ell}\left(\rho_{p^{2}}\right): \mathbb{Q}_{\ell}\left(\rho_{p}\right)\right]=1$ for almost all $\ell$, then the polynomial $x^{p^{2}}-1$ is a product of linear polynomials over $\mathbb{Q}_{\ell}\left(\rho_{p}\right)$ for almost all $\ell$. Then by [2, Exercise 6, p. 362], the polynomial $x^{p^{2}}-1$ is a product of linear polynomials over $\mathbb{Q}\left(\rho_{p}\right)$. But this is not the case since $\left[\mathbb{Q}\left(\rho_{p^{2}}\right): \mathbb{Q}\left(\rho_{p}\right)\right]=p$. Hence $\left[\mathbb{Q}_{\ell}\left(\rho_{p^{2}}\right): \mathbb{Q}_{\ell}\left(\rho_{p}\right)\right]=p$ for infinitely many $\ell$. For any such $\ell$, setting $K=$ $\mathbb{Q}_{\ell}\left(\rho_{p}\right)$ we have $\rho_{p^{2}} \notin K^{*}, \rho_{p} \in K^{*}$.

Remark 3.14. Note that algebras from Propositions 3.12 and 3.13 have bad local invariants (in the sense of Definition [1.5). Indeed, every constant algebra $\mathcal{C}$ of exponent 2 (resp. $p$ ) splits over $F(\sqrt{\alpha})$ (resp. $F\left(\sqrt[p]{\alpha^{s} \pi}\right)$ ). Hence in the case of exponent 2 we have $\mathcal{A} \otimes \mathcal{C} \otimes F(\sqrt{\alpha}) \sim \mathcal{A} \otimes F(\sqrt{\alpha})$, i.e. $\operatorname{ind}(\mathcal{A} \otimes \mathcal{C} \otimes F(\sqrt{\alpha}))=$ $\operatorname{ind}(\mathcal{A} \otimes F(\sqrt{\alpha}))=4$, and $\mathcal{A}$ has bad local invariants. The case of exponent $p$ is similar.

We now study good collections of local invariants over $K(t)$, often restricting our attention to algebras with linear ramification. Note that in the following proposition 
we describe the indices of all algebras of exponent 2 with given invariants (and not just their Faddeev indices).

Proposition 3.15. Let $\mathcal{A}$ be a central simple $F$-algebra of exponent 2 , and suppose that $\mathcal{A}$ is ramified only at three linear points $\left(t-a_{i}\right), i=1,2,3$. Then under a suitable numeration of the $a_{i}$ 's, $\mathcal{A}$ is Faddeev equivalent to the algebra

$$
\mathcal{B}=\left(\alpha,\left(t-a_{1}\right)\left(t-a_{2}\right)\right) \otimes\left(\pi,\left(t-a_{2}\right)\left(t-a_{3}\right)\right) .
$$

Moreover, any algebra of exponent 2 Faddeev equivalent to $\mathcal{A}$ is Brauer equivalent to one of the algebras

$$
\mathcal{B}(u)=\left(\alpha,\left(t-a_{1}\right)\left(t-a_{2}\right)\right) \otimes\left(\pi, u\left(t-a_{2}\right)\left(t-a_{3}\right)\right)
$$

with $u \in\{1, \alpha\}$.

Suppose in addition that $\bar{a}_{i} \neq \bar{a}_{j}, i \neq j$. Then the algebra $\mathcal{B}(u)$ has index 4 if and only if $u\left(a_{1}-a_{2}\right)\left(a_{1}-a_{3}\right) \in\left(K^{*}\right)^{2}$.

Proof. The algebra

$$
\mathcal{C}=\left(b_{1}, t-a_{1}\right) \otimes\left(b_{2}, t-a_{2}\right) \otimes\left(b_{3}, t-a_{3}\right)
$$

has prescribed ramification at $t-a_{1}, t-a_{2}$ and $t-a_{3}$. By the reciprocity law, $\mathcal{C}$ and $\mathcal{A}$ have the same ramification at $\infty$.

Then $\mathcal{C}$ is unramified at infinity since $\mathcal{A}$ is unramified at infinity. Hence $b_{1} b_{2} b_{3} \in$ $\left(K^{*}\right)^{2}$. Since $b_{i} \notin\left(K^{*}\right)^{2}, i=1,2,3$, then under a suitable numeration one can assume $b_{1}=\alpha, b_{2}=\alpha \pi, b_{3}=\pi$. Therefore

$$
\begin{aligned}
\mathcal{C} & =\left(\alpha, t-a_{1}\right) \otimes\left(\alpha \pi, t-a_{2}\right) \otimes\left(\pi, t-a_{3}\right) \\
& \sim\left(\alpha,\left(t-a_{1}\right)\left(t-a_{2}\right)\right) \otimes\left(\pi,\left(t-a_{2}\right)\left(t-a_{3}\right)\right)=\mathcal{B} .
\end{aligned}
$$

Hence $\mathcal{A}$ is Faddeev equivalent to $\mathcal{B}$.

Since the 2-torsion of $\operatorname{Br}(K)$ is generated by the class containing $(\alpha, \pi)$, we conclude that $\mathcal{A}$ is Brauer equivalent to $\mathcal{B}=\mathcal{B}(1)$ or to $\mathcal{B} \otimes(\alpha, \pi)=\mathcal{B}(\alpha)$.

The algebra $\mathcal{B}(u)$ has index 4 if and only if the Albert form

$$
\begin{aligned}
& \left\langle\alpha,\left(t-a_{1}\right)\left(t-a_{2}\right),-\alpha\left(t-a_{1}\right)\left(t-a_{2}\right),\right. \\
& \left.\quad-\pi,-u\left(t-a_{2}\right)\left(t-a_{3}\right), \pi u\left(t-a_{2}\right)\left(t-a_{3}\right)\right\rangle
\end{aligned}
$$

is anisotropic over $F$. By Springer's theorem, this form is anisotropic if the forms $\left\langle-1, \bar{u}\left(t-\bar{a}_{2}\right)\left(t-\bar{a}_{3}\right)\right\rangle$ and

$$
\left\langle\bar{\alpha},\left(t-\bar{a}_{1}\right)\left(t-\bar{a}_{2}\right),-\bar{\alpha}\left(t-\bar{a}_{1}\right)\left(t-\bar{a}_{2}\right),-\bar{u}\left(t-\bar{a}_{2}\right)\left(t-\bar{a}_{3}\right)\right\rangle
$$

are anisotropic over $k(t)$. The first form is anisotropic since $\bar{a}_{2} \neq \bar{a}_{3}$. As to the second form, by Springer's theorem it is anisotropic if the forms

$$
\left\langle\bar{\alpha},-\bar{u}\left(\bar{a}_{1}-\bar{a}_{2}\right)\left(\bar{a}_{1}-\bar{a}_{3}\right)\right\rangle \quad \text { and }\langle 1,-\bar{\alpha}\rangle
$$

are anisotropic, or, equivalently, if $\bar{u}\left(\bar{a}_{1}-\bar{a}_{2}\right)\left(\bar{a}_{1}-\bar{a}_{3}\right) \in\left(k^{*}\right)^{2}$. Hence $\mathcal{B}(u)$ has index 4 provided $u\left(a_{1}-a_{2}\right)\left(a_{1}-a_{3}\right) \in\left(K^{*}\right)^{2}$.

Assume now that $u\left(a_{1}-a_{2}\right)\left(a_{1}-a_{3}\right) \notin\left(K^{*}\right)^{2}$.

Let $L=F\left(\sqrt{u\left(t-a_{2}\right)\left(t-a_{3}\right)}\right)$. Note that the extension $L / K$ is purely transcendental. Indeed,

$$
K(t)=K\left(u\left(1+\left(a_{3}-a_{2}\right) /\left(t-a_{3}\right)\right)\right)=K\left(u\left(t-a_{2}\right) /\left(t-a_{3}\right)\right) .
$$

Then one can set

$$
z^{2}=u\left(t-a_{2}\right) /\left(t-a_{3}\right)
$$


and see that $L=K\left(\sqrt{u\left(t-a_{2}\right) /\left(t-a_{3}\right)}\right)=K(z)$. Then

$$
\mathcal{B}(u) \otimes_{F} L \sim\left(\alpha,\left(t-a_{1}\right)\left(t-a_{2}\right)\right) \otimes_{F} L \sim\left(\alpha, u\left(t-a_{1}\right)\left(t-a_{3}\right)\right) \otimes_{F} L .
$$

From the two expressions for $\mathcal{B}(u) \otimes_{F} L$, it is clear that $\mathcal{B}(u) \otimes_{F} L$ does not ramify at any point above $t-a_{2}$ or $t-a_{3}$ or $\infty$ since $\left(\alpha,\left(t-a_{1}\right)\left(t-a_{2}\right)\right)$ does not ramify at $t-a_{3}$ or $\infty$ and $\left(\alpha, u\left(t-a_{1}\right)\left(t-a_{3}\right)\right)$ does not ramify at $t-a_{2}$ or $\infty$. Thus it can only ramify above $t-a_{1}$, and there is only one such point. The ramification of $\mathcal{B}(u)$ at $t-a_{1}$ is given by $K(\sqrt{\alpha}) / K$. Since the residue field at the point of $L$ over $t-a_{1}$ is $K(\sqrt{\alpha})$, then $\mathcal{B}(u) \otimes_{F} L$ does not ramify at the point above $t-a_{1}$. Thus $\mathcal{B}(u) \otimes_{F} L$ ramifies at no points.

Note that the point above $t-a_{3}$ is $K$-rational. Let $L_{t-a_{3}}$ be the completion of $L$ with respect to the valuation extending the valuation of $F$ defined by $t-a_{3}$. Furthermore,

$$
\begin{aligned}
\mathcal{B}(u) \otimes_{F} L_{t-a_{3}} & \sim\left(\alpha,\left(t-a_{1}\right)\left(t-a_{2}\right)\right) \otimes_{F} L_{t-a_{3}} \\
& \sim\left(\alpha,\left(a_{3}-a_{1}\right)\left(a_{3}-a_{2}\right)\right) \otimes_{F} L_{t-a_{3}} .
\end{aligned}
$$

Since $\bar{a}_{i} \neq \bar{a}_{j}, i \neq j$, we conclude that $\left(a_{3}-a_{1}\right)\left(a_{3}-a_{2}\right)$ is a unit. Hence

$$
\left(\alpha,\left(a_{3}-a_{1}\right)\left(a_{3}-a_{2}\right)\right) \sim 1 \text { and } \mathcal{B}(u) \otimes_{F} L_{t-a_{3}} \sim 1 .
$$

Thus the algebra $\mathcal{B}(u) \otimes_{F} L$ is trivial (because it is unramified, and the completion $\mathcal{B}(u) \otimes_{F} L_{t-a_{3}}$ at a $K$-rational point is trivial), and therefore $\mathcal{B}(u)$ cannot have index 4. The proposition is proved.

In the case where $K$ is an $\ell$-adic field with residue field of characteristic $\neq 2$, Corollary 2.4 can be formulated without assuming the existence of a $K$-rational ramification point. This is because the case where none of three (geometric) ramification points is $K$-rational does not occur.

Indeed, assume that there exists an algebra $\mathcal{A}$ of exponent 2 with ramification only at an irreducible polynomial $f$ of degree 3 . Let $\theta$ be a root of $f$.

First consider the case where the extension $K(\theta) / K$ is cyclic. Then $\mathcal{A} \otimes_{F} F(\theta)$ ramifies at three linear points $t-a, t-b$ and $t-c$, where $f(t)=(t-a)(t-b)(t-c)$.

The algebra $\mathcal{A}$ can be written in the form

$$
(g(t), f(t)) \otimes\left(\otimes\left(h_{i}(t), h_{j}(t)\right)\right)
$$

where the polynomials $\left.g(t), h_{i}(t), h_{j}(t)\right)$ are relatively prime to $f(t)$. Then the ramifications of $\mathcal{A} \otimes F(\theta)$ at the points $t-a, t-b, t-c$ are $F(\theta)(\sqrt[p]{g(a)}) / F(\theta)$, $F(\theta)(\sqrt[p]{g(b)}) / F(\theta), F(\theta)(\sqrt[p]{g(c)}) / F(\theta)$, respectively. Let $v=g(a)$; then $g(b)=$ $\sigma(v), g(c)=\sigma^{2}(v)$.

Then the algebra $\mathcal{A} \otimes_{F} F(\theta)$ is Faddeev equivalent to the algebra

$$
\mathcal{B}=(v, t-a) \otimes_{F(\theta)}(\sigma(v), t-b) \otimes_{F(\theta)}\left(\sigma^{2}(v), t-c\right)
$$

for some $v \in K(\theta)^{*}$, where $\sigma$ is a generator of $\operatorname{Gal}(K(\theta) / K)$.

Indeed, $\mathcal{B}$ has prescribed ramification at $t-a, t-b$ and $t-c$. By the reciprocity law, $\mathcal{B}$ and $\mathcal{A} \otimes_{F} F(\theta)$ have the same ramification at $\infty$.

The algebra $\mathcal{B}$ is unramified at infinity since $\mathcal{A}$ is unramified at infinity. Hence we have $N_{K(\theta) / K}(v)=v \sigma(v) \sigma^{2}(v) \in\left(K^{*}\right)^{2}$. Since $\operatorname{char}(k) \neq 2$, the map $N_{K(\theta) / K}$ induces an isomorphism of the groups $K(\theta)^{*} /\left(K(\theta)^{*}\right)^{2}$ and $K^{*} /\left(K^{*}\right)^{2}$ (it is a surjective map of finite groups of equal order). Hence $v \in\left(K(\theta)^{*}\right)^{2}$, and $\mathcal{A} \otimes_{F} F(\theta)$ is 
unramified. But this is impossible since $\mathcal{A}$ ramifies at $f$ and the degree of the extension $K(\theta) / K$ is 3 . Thus $\mathcal{A}$ cannot ramify only at an irreducible cubic polynomial $f$.

Now consider the case when $K(\theta) / K$ is not cyclic. Let $d$ be the discriminant of $f$. Then $f$ is irreducible over $K(\sqrt{d}) / K$, and $\mathcal{A} \otimes_{F} F(\sqrt{d})$ can ramify only at $f$. The extension $K(\sqrt{d})(\theta) / K(\sqrt{d})$ is cyclic, and as proved before, the algebra $\mathcal{A} \otimes_{F} F(\sqrt{d})$ cannot ramify at $f$. Thus $\mathcal{A} \otimes_{F} F(\sqrt{d})$ is unramified, and $\mathcal{A}$ is Faddeev equivalent to $(d, f)$. Since $(d, f)$ ramifies at infinity, so does $\mathcal{A}$, a contradiction. This shows that $\mathcal{A}$ cannot ramify only at an irreducible polynomial $f$ of degree 3 .

We have proved the following

Proposition 3.16. If char $(k) \neq 2, F=K(t)$, and $\mathcal{A}$ is a central simple $F$-algebra of exponent 2 with (geometric) ramification at three points, then $\mathcal{A}$ is Faddeev equivalent to a quaternion F-algebra.

\section{Number Field CASE}

In this section we make a few remarks about the number field case focusing on "Hasse principles". As usual, we denote $F=K(t)$.

Note that if an algebra $\mathcal{A}$ over $F$ is Faddeev equivalent to a quaternion algebra, then $\mathcal{A} \sim \mathcal{C} \otimes \mathcal{B}$, where $\mathcal{C}$ is a quaternion $K$-algebra and $\mathcal{B}$ is a quaternion $F$-algebra. Hence without loss of generality we can restrict our attention to algebras of index at most 4. Namely one can pose the following natural questions.

Question 4.1. Let $K$ be a number field, let $F=K(t)$, and let $\mathcal{A}$ be a central simple $F$-algebra of exponent 2. Suppose that the indices of all $K_{v}(t)$-algebras $\mathcal{A} \otimes_{F} K_{v}(t)$ do not exceed 2 (here $v$ runs over all nonequivalent valuations of $K$ ). Is it true that the index of $\mathcal{A}$ does not exceed 2 ?

Question 4.2. Let $K$ be a number field, let $F=K(t)$, and let $\mathcal{A}$ be a central simple $F$-algebra of exponent 2. Suppose that the Faddeev indices of all $K_{v}(t)$ algebras $\mathcal{A} \otimes_{F} K_{v}(t)$ do not exceed 2. Is it true that the Faddeev index of $\mathcal{A}$ does not exceed 2 ?

It turns out that neither of these "Hasse principles" holds.

Proposition 4.3. Let $K=\mathbb{Q}, \mathcal{A}=(17, t) \otimes(13,(t-1)(t-11))$. Then the Faddeev index of $\mathcal{A}$ equals 4 but the indices of $\mathcal{A} \otimes_{F} K_{v}(t)$ do not exceed 2.

Proof. Consider the algebra $\mathcal{B}=(13,11) \otimes \mathbb{Q}(\sqrt{17})$. Since $17 \in\left(\mathbb{Q}_{13}^{*}\right)^{2}$, we have

$$
\mathcal{B} \otimes \mathbb{Q}_{13}(\sqrt{17}) \sim(13,11) \otimes \mathbb{Q}_{13} .
$$

Since $11 \notin\left(\mathbb{Q}_{13}^{*}\right)^{2}$, the latter algebra is not trivial. Thus $\mathcal{B}$ is not trivial. Then by Proposition 2.7 the algebra $\mathcal{A}$ has Faddeev index 4.

On the other hand, since 17 and 13 are units in $\mathbb{Q}_{\ell}$ for $\ell \neq 13,17$, the algebra $\mathcal{A} \otimes \mathbb{Q}_{\ell}$ has index less than 4 for $l \neq 13,17$. Since $17 \in\left(\mathbb{Q}_{13}^{*}\right)^{2}$ and $13 \in\left(\mathbb{Q}_{17}^{*}\right)^{2}$ $(8 \cdot 8=13+17 \cdot 3)$, the algebras $\mathcal{A} \otimes \mathbb{Q}_{13}$ and $\mathcal{A} \otimes \mathbb{Q}_{17}$ have index less than 4 .

Our next goal is to explicitly construct algebras over $\mathbb{Q}(t)$ with Faddeev index greater than 2 (bad systems of invariants). Let us try to use Proposition 2.7 in order to obtain a sufficient condition for an algebra over $\mathbb{Q}(t)$ to have Faddeev index not equal to 2. We keep the notation of Proposition 2.7. We may (and will) assume that $a, b$ and $c$ are square-free integers. 
If $c<0$ and $a b<0$, then one of the algebras from Proposition 2.7 is not trivial, and the corresponding invariants are bad.

Assume that there is a prime $p$ such that $p \nmid b c, p \mid a$, and the Legendre symbols $\left(\frac{b}{p}\right)$ and $\left(\frac{c}{p}\right)$ satisfy

$$
\left(\frac{b}{p}\right)=1, \quad\left(\frac{c}{p}\right)=-1 .
$$

Then $((a, c) / \mathbb{Q}(\sqrt{b})) \otimes \mathbb{Q}_{p} \nsim 1$. Hence $((a, c) / \mathbb{Q}(\sqrt{b})) \nsim 1$, and the corresponding system of invariants is bad.

The case where there is a prime $p$ such that $p \nmid a c, p \mid b$, and

$$
\left(\frac{a}{p}\right)=1, \quad\left(\frac{c}{p}\right)=-1,
$$

is similar.

Assume that there is a prime $p$ such that $p \nmid a b, p \mid c$, and either

$$
\left(\frac{b}{p}\right)=1, \quad\left(\frac{a}{p}\right)=-1
$$

or

$$
\left(\frac{a}{p}\right)=1, \quad\left(\frac{b}{p}\right)=-1 .
$$

Then either $((a, c) / \mathbb{Q}(\sqrt{b})) \otimes \mathbb{Q}_{p} \nsim 1$ or $((b, c) / \mathbb{Q}(\sqrt{a})) \otimes \mathbb{Q}_{p} \nsim 1$, and the corresponding system of invariants is bad.

Finally, if there is a prime $p$ such that $p \nmid a, p \mid b$, and $p \mid c$, set $c=p c_{p}, b=p b_{p}$. If

$$
\left(\frac{a}{p}\right)=1, \quad\left(\frac{-b_{p} c_{p}}{p}\right)=-1,
$$

then the corresponding system of invariants is bad. The case where $p \nmid b, p|a, p| c$ is similar.

Thus we have proved the following

Proposition 4.4. Let $\mathcal{A}$ be an algebra over $F$ with ramification $K(\sqrt{a}) / K$ at $t, \infty$ and $K(\sqrt{b}) / K$ at $t-1, t-c$. Then any of the following conditions is sufficient for $\mathcal{A}$ to have Faddeev index 4 :

1. $c<0$ and $a b<0$;

2. $p \nmid b c, p \mid a$, and the Legendre symbols $\left(\frac{b}{p}\right)$ and $\left(\frac{c}{p}\right)$ satisfy

$$
\left(\frac{b}{p}\right)=1, \quad\left(\frac{c}{p}\right)=-1 .
$$

3. $p \nmid a c, p \mid b$, and

$$
\left(\frac{a}{p}\right)=1, \quad\left(\frac{c}{p}\right)=-1 .
$$

4. $p \nmid a b, p \mid c$, and either

$$
\left(\frac{b}{p}\right)=1, \quad\left(\frac{a}{p}\right)=-1
$$

or

$$
\left(\frac{a}{p}\right)=1, \quad\left(\frac{b}{p}\right)=-1 .
$$

Let us now prove an analog of Proposition 3.16. 
Proposition 4.5. If $\mathcal{A}$ is a central simple $F$-algebra of exponent 2 with (geometric) ramification at three points, then $\mathcal{A}$ is Faddeev equivalent to a quaternion $F$-algebra.

Proof. In light of Corollary 2.4, it suffices to prove that an algebra $\mathcal{A}$ of exponent 2 cannot ramify only at an irreducible polynomial $f$ of degree 3 . Let $\theta$ be a root of such an $f$.

As in the proof of Proposition 3.16, we may (and will) assume that the extension $\mathbb{Q}(\theta) / \mathbb{Q}$ is cyclic. It is enough to show that there is no $v \in \mathbb{Q}(\theta)^{*}$ such that $N_{\mathbb{Q}(\theta) / \mathbb{Q}}(v) \in\left(\mathbb{Q}^{*}\right)^{2}$ and $v \notin\left(\mathbb{Q}(\theta)^{*}\right)^{2}$.

Assume that such a $v$ exists. By the Chebotarev density theorem, there is a prime $p \neq 2$ such that $f$ is irreducible over $\mathbb{Q}_{p}$ and $v \notin\left(\mathbb{Q}_{p}(\theta)^{*}\right)^{2}$. Hence $N_{\mathbb{Q}_{p}(\theta) / \mathbb{Q}_{p}}(v) \in\left(\mathbb{Q}_{p}^{*}\right)^{2}$. But this is impossible (see the proof of Proposition 3.16).

Let $\mathcal{A}$ be a central simple $F$-algebra of exponent 2. Suppose that $\mathcal{A}$ has ramification at $m$ linear points $t-a_{i}, i=1, \ldots, m$, and there is a prime $\ell$ such that all residues $\bar{a}_{i} \bmod \ell$ are pairwise distinct. Then by Proposition $3.2, \mathcal{A} \otimes_{\mathbb{Q}(t)} \mathbb{Q}_{\ell}(t)$ is Faddeev equivalent to a tensor product

$$
\mathcal{B}=(\alpha, f) \otimes_{\mathbb{Q}_{\ell}(t)}(\pi, u g),
$$

where $u \in R^{*}$, and $f, g$ are products of some of the $t-a_{i}$.

Proposition 4.6. With the above notation, assume that $\operatorname{deg} g$ is odd. Suppose that there are roots $b_{1}, b_{2}$ of $f$ such that $g\left(b_{1}\right), \alpha g\left(b_{2}\right) \notin\left(\mathbb{Q}_{\ell}^{*}\right)^{2}$. Then $\mathcal{A}$ is not Faddeev equivalent to a quaternion algebra.

Proof. Follows from Proposition 3.10 .

\section{Pencils of Cyclic Severi-Brauer varieties}

In this section we present the geometric counterpart of the above algebraic considerations. Throughout below $K$ is assumed to be a field of characteristic zero. Let us first define our main object.

Definition 5.1 (9). Let $n \geq 2$ be an integer. A pencil of Severi-Brauer varieties is a smooth, projective, geometrically integral $K$-variety $X$ admitting a dominant $K$-morphism $\varphi: X \rightarrow \mathbb{P}_{K}^{1}$ whose generic fiber $X_{\eta}$ is a Severi-Brauer variety $V$ of dimension $n-1$.

Recall that $V$ is defined as an $F$-form of $\mathbb{P}^{n-1}$, i.e. $V \times_{F} L \cong \mathbb{P}_{L}^{n-1}$ for some extension $L / F$; here $F$ denotes $K(t)$. It is well known that there is a one-to-one correspondence between Severi-Brauer $F$-varieties $V$ of dimension $n-1$ and central simple $F$-algebras $\mathcal{A}$ of rank $n^{2}$. Let us focus on the following special case.

Definition 5.2. We call $X$ a pencil of cyclic Severi-Brauer varieties if its generic fiber corresponds to a cyclic $F$-algebra.

For simplicity, further on we only consider the case $n=p$, a prime number. Note that if $n=2, X$ is none other than a conic bundle surface. We want to study a problem of construction of $X$ with prescribed degeneration data. The points of degeneration of the fibration $\varphi$ are precisely the closed points $x \in \mathbb{P}^{1}$, where the residues $\partial_{x}(\mathcal{A})$ are nonzero (this observation allows us to relate geometric problems considered in this section to results of Sections 24). The geometric structure of the degenerate fibers of $\varphi$ is described in [3, 15]: Over a geometric point $\bar{x}$ the degenerate fiber consists of $p$ components $\bar{V}_{1}, \ldots, \bar{V}_{p}$ intersecting transversally; see 
the papers cited above for more details. For $p=2$, such a fiber is a union of two projective lines with normal crossing; see [16], 17]. For $p=3$, each $\bar{V}_{i}$ is the rational scroll $F_{1}$ (= a ruled surface obtained by blowing up a point in $\mathbb{P}^{2}$ ); on $F_{1}$ there is the exceptional curve $E$ and the ruling $C ; \bar{V}_{i}$ and $\bar{V}_{i+1}(i \in \mathbb{Z} / 3)$ intersect along $C_{i}$ and $E_{i+1}$, there are 3 isolated points on each degenerate fiber (see [3, 1.5]). Below we assume that $X$ is relatively minimal, that is, one cannot blow down any component of any degenerate fiber.

First let us give a straightforward generalization of Question C.

Problem 5.3. Let $\left\{x_{i}\right\}_{1 \leq i \leq s}$ be a finite collection of closed points of $\mathbb{P}_{K}^{1}$. Let ${ }_{p} \operatorname{Br}(K)$ be the $p$-torsion subgroup of $\operatorname{Br}(K)$, and let

$$
\partial:{ }_{p} \operatorname{Br}(K) \rightarrow \bigoplus_{i=1}^{s} H^{1}\left(K\left(x_{i}\right), \mathbb{Z} / p\right)
$$

be the residue map. Let $\left\{d_{i}\right\}_{1 \leq i \leq s} \in \operatorname{im} \partial$. Does there exist over $F$ a pencil $X$ of Severi-Brauer varieties of dimension $p-1$ with local invariants $\left\{x_{i}, d_{i}\right\}$ ? Does there exist a pencil of cyclic Severi-Brauer varieties?

A translation of this question into the language of algebras immediately leads to a negative answer to Problem 5.3 for some $K$ : indeed, we can use the results of Section 3 to obtain

Theorem 5.4. With the notation of Section 3 , let $\mathcal{A}$ be as in Theorem 3.6 or Proposition 3.13 , and let $\left\{x_{i}, d_{i}\right\}$ be the collection of its local invariants. Then there is no pencil of Severi-Brauer varieties with these local invariants.

We now go over to another setting of the above problem with a goal to obtain some positive results. It turns out that if we are allowed to move ramification points along $\mathbb{P}^{1}$, the answer will always be positive. Moreover, under this setting we can even get a pencil of cyclic Severi-Brauer varieties with prescribed Galois action on degenerate fibers. The following is a generalization of the conic bundle case [22].

Let $G=\operatorname{Gal}(\bar{K} / K)$ denote the Galois group. Suppose that a pencil $X$ degenerates at closed points $x_{i}, 1 \leq i \leq s$, and denote $r=\sum_{i=1}^{s} \operatorname{deg}\left(x_{i}\right)$. Then our pencil has $r$ geometric degenerate fibers whose components we designate as $C_{i}^{j}(1 \leq i \leq r, 1 \leq j \leq p)$. The Galois action respects degenerate fibers and intersection indices, so we obtain a representation $\lambda: G \rightarrow \operatorname{Perm}\left(C_{i}^{j}\right)=\left(S_{p}\right)^{r} \rtimes S_{r}$, where $S_{m}$ is the symmetric group on $m$ letters and $\rtimes$ stands for the semidirect product.

Definition 5.5. With the above notation, we call $G_{X}=\operatorname{im}(\lambda)$ the splitting group of $X$.

Of course, the nature of the splitting group is related to the number and the degrees of the ramification points of the corresponding algebra. Let $K_{i}=K\left(x_{i}\right)$ denote the residue field at $x_{i}$, and let $L_{i} / K_{i}$ be the cyclic extension of degree $p$ defined by the local invariant of the corresponding algebra. Let $L$ be the Galois closure of the compositum of the fields $L_{i}$ in $\bar{K}$. Since all the components of the degenerate fibers of $X$ are defined over $L$, the subgroup $\operatorname{Gal}(\bar{K} / L)$ acts trivially on the set of those components (cf. [15, Prop. 2.3]), and $G_{X}$ is thus isomorphic to $G / \operatorname{ker}(\lambda)=G / \operatorname{Gal}(\bar{K} / L)=\operatorname{Gal}(L / K)$. We call $L$ the splitting field of $X$. Here are some simple (but typical) examples. 
Example 5.6. a) Let $X$ be a conic bundle surface given by an affine equation in $\mathbb{A}_{K}^{3}: y^{2}-b z^{2}=f(t)$, where $b$ is a nonsquare element of $K$. If $f(t)=$ $\left(t-a_{1}\right) \ldots\left(t-a_{2 n}\right)$ is a monic polynomial of even degree all of whose roots belong to $K$, then the corresponding algebra $\mathcal{A}=(b, f(t))$ is unramified at infinity; its local invariants at the finite ramification points $t-a_{i}$ are $K(\sqrt{b}) / K$. The fibration $X \rightarrow \mathbb{P}_{K}^{1}$ degenerates at $t=a_{i}$, and the components of each degenerate fiber are defined over $K(\sqrt{b})$. Hence $G_{X} \cong \mathbb{Z} / 2$ acts by interchanging the components of each fiber.

More generally, if $f(t)$ is an arbitrary polynomial of even degree, we have $G_{X} \cong$ $\mathbb{Z} / 2 \times \operatorname{Gal}\left(L_{f} / K\right)$, where $L_{f}$ is the splitting field of $f$ (see [21, Lemma 6.1]).

b) If $X$ is given by the equation $y^{2}-t z^{2}=f(t)$ where $f(t)=a_{0} t^{m}+\cdots+a_{m}$, then the corresponding algebra $\mathcal{A}=(t, f(t))$ may also ramify at infinity and at $t=0$, its local invariants being $K\left(\sqrt{a_{m}}\right) / K$ and $K\left(\sqrt{a_{0}}\right) / K$, respectively. If $a_{0}$ and $a_{m}$ are squares in $K$, then $G_{X}$ can be identified with $\operatorname{Gal}\left(L_{g} / K\right)$ where $g(t)=f\left(t^{2}\right)$ (see [21, Lemma 6.2]).

Our first observation concerning the splitting groups is as follows: $G_{X}$ lies in $\Gamma=\left(\mathbb{Z}_{p}\right)^{r} \rtimes S_{r}$. Indeed, since Gal $\left(\bar{K} / L_{i}\right)$ acts trivially on the $i$ th degenerate fiber, $G$ acts on it through $\mathrm{Gal}\left(L_{i} / K\right)$, and thus only cyclic subgroups of $S_{p}$ (corresponding to $\left.\operatorname{Gal}\left(L_{i} / K_{i}\right)\right)$ can appear as subgroups of $G_{X}$.

Using the Faddeev reciprocity law, one can say a bit more about $G_{X}$. For each $1 \leq i \leq r$, let us fix a generator $c_{i}$ of the cyclic subgroup $\mathbb{Z}_{p} \subset \Gamma$ permuting the components of the $i$ th degenerate geometric fiber.

Lemma 5.7. Let $W \subset \Gamma$ be the subgroup of index $p$ consisting of the elements of the form $c_{i_{1}}^{m_{1}} \ldots c_{i_{k}}^{m_{k}} \sigma$ with $m_{1}+\cdots+m_{k}$ divisible by $p$ and $\sigma \in S_{r}$. Then $G_{X} \subseteq W$.

Proof. Let $X$ degenerate at the points $x_{i} \in \mathbb{P}^{1}$ (which are not assumed rational), $1 \leq i \leq s$, and let $d_{i} \in H^{1}\left(K\left(x_{i}\right), \mathbb{Z} / p\right)$ be the residue at $x_{i}$. As in Proposition 1.9(ii), $d_{i}$ defines a cyclic extension $L_{i} / K\left(x_{i}\right)$ of degree $p$ together with the choice of a generator $h_{i}$ of $\operatorname{Gal}\left(L_{i} / K\left(x_{i}\right)\right)$ (cf. [30, Ch. XIV, $\left.\S 1\right]$ ). Denote by $\chi_{i}$ the character of $\operatorname{Gal}\left(K\left(x_{i}\right)\right)$ corresponding to $d_{i}$. We make our choice so that $\chi_{i}\left(h_{i}\right)=1$.

Let us first consider the simplest case where all $x_{i}$ 's belong to $K$. In this case we have $\operatorname{deg}\left(x_{i}\right)=1$ for all $i, K_{i}=K, s=r$, all corestriction maps are identities, and $G_{X}$ obviously lies in $\left(\mathbb{Z}_{p}\right)^{r}$, the subgroup acting on the components of each fiber.

We have to prove that for any $g \in \operatorname{Gal}(\bar{K} / K)$ the image $\lambda(g)$ lies in $\left(\mathbb{Z}_{p}\right)^{r-1}$, the index $p$ subgroup of $\left(\mathbb{Z}_{p}\right)^{r}$ mentioned in the lemma. Let $\lambda(g)=\prod_{i=1}^{r} c_{i}^{m_{i}}$. Denote by $g_{i} \in \operatorname{Gal}\left(L_{i} / K\right)$ the image of $g$ under the natural projection $\operatorname{Gal}(\bar{K} / K) \rightarrow$ $\operatorname{Gal}\left(L_{i} / K\right)$. We have $g_{i}=h_{i}^{m_{i}}$. According to our choice of $\chi_{i}, c_{i}$, we have $\chi_{i}(g)=$ $\chi_{i}\left(g_{i}\right)=\chi_{i}\left(h_{i}^{m_{i}}\right)=m_{i}$. By the Faddeev reciprocity law, we get $\sum_{i=1}^{r} m_{i}=0$, as required.

In the general case, for each $x_{i}$ denote by $\bar{x}_{i k}, 1 \leq k \leq \operatorname{deg}\left(x_{i}\right)$, the geometric degeneration points lying above $x_{i}$. Take $g \in \operatorname{Gal}(L / K)$ and denote $\lambda(g)=$ $\sigma \prod_{i=1}^{r} \prod_{k=1}^{\operatorname{deg}\left(x_{i}\right)} c_{i k}^{m_{i k}}$, where $\sigma \in S_{r}$ acts by permuting degenerate fibers as a whole, not touching the components of any fiber. Denote $\theta_{i}=\operatorname{cor}\left(\chi_{i}\right) \in H^{1}\left(L_{i} / K, \mathbb{Z} / p\right)$. According to the definition of the corestriction map, we have $\theta_{i}(g)=\sum_{k=1}^{\operatorname{deg}\left(x_{i}\right)} m_{i k}$. As in the previously considered particular case, the Faddeev reciprocity law gives the required assertion.

The lemma is proved. 
Remark 5.8. See [22] for a geometric proof of Lemma 5.7] in the case $p=2$.

One can now pose the following

Problem 5.9. Let $W$ be the subgroup of index $p$ in $\Gamma$ as above, and let $G$ be a subgroup of $W$. Does there exist a pencil $X$ of cyclic Severi-Brauer varieties defined over $K$, of dimension $p-1$ with splitting group $G_{X} \cong G$ ?

It turns out that Problem 5.9 has a positive solution under very mild assumptions on $K$.

Theorem 5.10. With the notation of Problem [5.9, suppose that there exists a Galois extension $L / K$ with group $G$. Assume that $K$ contains $\rho$, a primitive $p$ th root of 1 . Then there exists a pencil $X$ of cyclic Severi-Brauer varieties of dimension $p-1$, defined over $K$, with splitting group $G_{X} \cong G$.

Before proving the theorem, let us introduce some more notation. Denote by $E$ a vector $K$-space of dimension $r$, and let us fix a $K$-basis $\left\{e_{1}, \ldots, e_{r}\right\}$ of $E$. The group $\Gamma=(\mathbb{Z} / p)^{r} \rtimes S_{r}=\left\langle c_{1}, \ldots, c_{r}\right\rangle \rtimes S_{r}$ acts on this basis as follows: $c_{i}$ multiplies $e_{i}$ by $\rho$, and $S_{r}$ permutes the elements of the basis. We need the following lemma (we thank the referee for providing a shorter proof).

Lemma 5.11. With the above notation, let $L / K$ be a Galois extension of fields with group $G \subseteq \Gamma$. Then one can choose elements $\alpha_{1}, \ldots, \alpha_{r}$ in $L$ such that:

(i) the elements of the set $Z=\left\{\rho^{i} \alpha_{j}\right\}_{0 \leq i \leq p-1,1 \leq j \leq r}$ are pairwise distinct;

(ii) the map $e_{j} \mapsto \alpha_{j}$ commutes with the action of $G$.

Proof of the lemma. Let us view $E$ and $L$ as $K[G]$-modules and let us compute $\operatorname{Hom}_{K[G]}(E, L)$. First consider $\operatorname{Hom}_{K}(E, L)$. Let us view it as a module over the trivial crossed product $C=L[G, 1]$ where $C$ acts as follows: $l g(f)(e)=$ $l\left(g\left(f\left(g^{-1} e\right)\right)\right)$ for $f \in \operatorname{Hom}_{K}(E, L)$. Since $C$ is a matrix algebra, as a $C$-module $\operatorname{Hom}_{K}(E, L)$ is a direct sum of $L$ 's. Hence $\operatorname{Hom}_{K[G]}(E, L)$ is a direct sum of $K$ 's.

Let $f_{1}, \ldots, f_{s} \in \operatorname{Hom}_{K[G]}(E, L)$ be any finite set of $G$-homomorphisms, and let $f=\sum_{m=1}^{s} \beta_{m} f_{m}$ where $\beta_{m} \in K$. Set $\alpha_{j}=f\left(e_{j}\right), j=1, \ldots, r$. The set $Z=\left\{\rho^{i} \alpha_{j}\right\}$ is as desired once we choose $\beta_{m}$ lying outside a finite number of hyperplanes. Such a choice is possible because $K$ is infinite.

Proof of the theorem. We shall construct a pencil with $r+2$ degenerate fibers. Among those, two fibers will have each of their components defined over $K$, and the Galois action on the remaining $r$ degenerate fibers will coincide with the prescribed one. Then, after blowing down two fibers, we get the desired pencil.

Let us choose $\alpha_{i} \in L$ as in Lemma 5.11, Set

$$
g(t)=(-1)^{r}\left(t-\alpha_{1}^{p}\right) \ldots\left(t-\alpha_{r}^{p}\right) .
$$

Since the coefficients of $g(t)$ are symmetric functions in $\alpha_{i}^{p}$ and hence are $G$ invariant, we conclude that $g(t) \in K[t]$. Let $\mathcal{A}=(t, g(t))_{p}$, a cyclic algebra over $F=K(t)$ with nontrivial local invariants at the divisors of $g(t)$. Note that the invariant at $t$ is trivial because $g(0)=\left(\alpha_{1} \ldots \alpha_{r}\right)^{p}$ is a $p$ th power of an element of $K$; indeed, $\alpha_{1} \ldots \alpha_{r} \in L^{G}=K$ because

$$
c_{i_{1}}^{m_{1}} \ldots c_{i_{k}}^{m_{k}} \sigma\left(\alpha_{1} \ldots \alpha_{r}\right)=\rho^{m_{1}+\cdots+m_{k}}\left(\alpha_{1} \ldots \alpha_{r}\right)=\alpha_{1} \ldots \alpha_{r}
$$


Therefore the degenerate fiber at $t=0$ can be blown down. The invariant at infinity is also trivial: we have

$$
(1 / t, g(1 / t)) \sim\left(t^{r-1},(-1)^{r}\left(1-t \alpha_{1}^{p}\right) \ldots\left(1-t \alpha_{r}^{p}\right)\right) ;
$$

if $r$ is odd, then this algebra is unramified at $t=0$, if $r$ is even, then its invariant at $t=0$ equals 1 . Hence the degenerate fiber at infinity can be also blown down.

Thus the pencil of Severi-Brauer varieties with generic fiber corresponding to $\mathcal{A}$ (with degenerate fibers at $t=0$ and $t=\infty$ blown down) degenerates only at $t=\alpha_{i}^{p}$. The corresponding residue fields are $K\left(\alpha_{i}^{p}\right)$; the components of the $i$ th degenerate fiber are defined over $K\left(\alpha_{i}^{p}\right)\left(\alpha_{i}\right)$, and we get the prescribed Galois data: $G_{X} \cong G$.

Remark 5.12. Theorem 5.10 is a generalization of [21, Th. 6.3], which solves, for a conic bundle surface, a problem of Manin 25] (still open in general): to construct a rational surface $X$ with given Galois module $\operatorname{Pic}(\bar{X})$.

\section{APPENDIX}

\section{CyClicity of ALGEBRAS OVER $k(x, y)$}

In this Appendix we apply our results to a situation studied by Ford [14, Van den Bergh [34, and Jacob [18, namely division algebras over $k(x, y)$, where $k$ is an algebraically closed field. We view $F=k(x, y)$ as $K(y)$, where $K=k(x)$. Since $k$ is algebraically closed, $\operatorname{Br}(K)=0$, so Faddeev and Brauer equivalence are the same. We use this fact repeatedly.

Let $k$ be an algebraically closed field, let $\mathbb{P}_{k}^{2}$ be the projective plane over $k$, and let $k(X, Y, Z) / k$ be a purely transcendental extension of degree 3 with basis $\{X, Y, Z\}$. Setting $x=X / Z, y=Y / Z$, one can regard $k(x, y) \subset k(X, Y, Z)$ as the field of rational functions on $\mathbb{P}_{k}^{2}$ and on the affine chart $\mathbb{A}_{k}^{2}$ defined by the condition $Z \neq 0$; here $(X: Y: Z)$ are homogeneous coordinates on $\mathbb{P}_{k}^{2}$ and $(x, y)$ are affine coordinates on $\mathbb{A}_{k}^{2}$.

On the other hand, since $k(x, y)=k(x)(y) \subset k(x)(Y, Z)$, one can interpret $k(x, y)$ as the field of rational functions on the projective line $\mathbb{P}_{k(x)}^{1}$ over $k(x)$ (with homogeneous coordinates $(Y: Z)$ ) and on the affine line $\mathbb{A}_{k(x)}^{1}$ defined by the condition $Z \neq 0$ (with affine coordinate $y=Y / Z$ ).

Following Jacob [18, we denote by $\mathbb{P}_{k}^{2(1)}$ (resp. $\left.\mathbb{P}_{k(x)}^{1(0)}\right)$ the set of codimension 1 points on $\mathbb{P}_{k}^{2}$ (resp. $\mathbb{P}_{k(x)}^{1}$ ). Elements of $\mathbb{P}_{k}^{2(1)}$ are in one-to-one correspondence with monic (with respect to the lexicographic ordering $X>Y>Z$ ), $k$-irreducible, positive degree forms in $X, Y, Z$, and elements of $\mathbb{P}_{k(x)}^{1(0)}$ are in one-to-one correspondence with monic $k(x)$-irreducible forms in $Y, Z$.

The point of $\mathbb{P}_{k}^{2(1)}$ given by the form $Z=0$ will be called infinite (or the infinite line), and all the others will be called finite. Clearly, finite codimension 1 points on the plane $\mathbb{P}_{k}^{2}$ (resp. the line $\mathbb{P}_{k(x)}^{1}$ ) are in bijective correspondence with monic $k$-irreducible polynomials in $x, y$ (resp. monic $k(x)$-irreducible polynomials in $y$ ) given by the rule

$$
F(X, Y, Z) \mapsto P(x, y)=F(X, Y, Z) / Z^{\operatorname{deg} F}
$$

(resp.

$$
\left.F(Y, Z) \mapsto P(y)=F(Y, Z) / Z^{\operatorname{deg} F}\right)
$$


Throughout below we use these bijections and sometimes only speak about the polynomials $P(x, y)$ and $P(y)$, for brevity calling them points.

To work with finite points, we have to throw away those points corresponding to the irreducible polynomials from $k[x, y]$ which depend only on $x$. Indeed, a valuation of $k(x, y)$ corresponding to such a polynomial remains nontrivial after restriction to $k(x)$ and thus does not induce any valuation of $k(x)(y)$ trivial on the "constant field" $k(x)$. We shall sum up this observation in the following simple lemma.

Lemma A 1. Let $\mathbb{A}_{k}^{2(1)}$ (resp. $\mathbb{A}_{k(x)}^{(0)}$ ) denote the set of finite codimension 1 points on $\mathbb{P}_{k}^{2}$ (resp. $\mathbb{P}_{k(x)}^{1}$ ). Let $S \subset \mathbb{A}_{k}^{2(1)}$ denote the set of points corresponding to the linear polynomials $x-a, a \in k$. There is a bijection $\pi: \mathbb{A}_{k}^{2(1)} \backslash S \rightarrow \mathbb{A}_{k(x)}^{1(0)}$ defined as follows: if $P(x, y)=\sum_{i=0}^{n} a_{i}(x) y^{i}$, then $\pi(P(x, y))=P(x, y) / a_{n}(x)$.

Proof. From the hypothesis of the lemma it follows that $\operatorname{deg}_{y}(\pi(P(x, y))>0$. If

$$
T(y)=y^{n}+\sum_{i=0}^{n-1} \frac{a_{i}(x)}{b_{i}(x)} y^{i} \in k(x)[y],
$$

where $a_{i}(x), b_{i}(x) \in k[x], \operatorname{deg}_{y} T>0$, we define $\pi^{\prime}(T(y))=F(x) T(y) / a$, where $F(x)$ is the least common multiple of the polynomials $b_{0}(x), \ldots, b_{n-1}(x)$ and $a$ stands for the leading coefficient of $F(x) T(y)$. Obviously, the map $\pi^{\prime}$ is inverse to $\pi$.

Let $R$ be a discrete valuation ring, let $K$ be the fraction field of $R$, and let $k(R)$ be the residue field. Assume $k$ to be of characteristic zero. There is an exact sequence [4]

$$
0 \rightarrow \operatorname{Br} R \rightarrow \operatorname{Br} K \stackrel{\partial_{R}}{\longrightarrow} \operatorname{Hom}_{\text {cont }}\left(G_{R}, \mathbb{Q} / \mathbb{Z}\right)=H^{1}\left(G_{R}, \mathbb{Q} / \mathbb{Z}\right),
$$

where $G_{R}$ is the absolute Galois group of $k(R)$.

We say that a $k(x, y)$-algebra $\mathcal{A}$ ramifies at an irreducible polynomial $F(x, y) \in$ $k[x, y]$ (resp. $F(y) \in k(x)[y]$ ) if $\partial_{R_{F}}([\mathcal{A}]) \neq 0$, where $R_{F}$ is the valuation ring corresponding to the curve given by $F(x, y)=0$ (resp. the valuation ring corresponding to $F(y) \in k(x)[y])$.

Lemma A 2. Suppose that $\operatorname{deg}_{y} F(x, y)>0$. Then the valuations of $k(x, y)$ corresponding to the polynomials $F(x, y) \in k[x, y]$ and $\pi(F(x, y)) \in k(x)[y]$ coincide.

Proof. Since the degree of $F(x, y)$ with respect to $y$ is positive, we have $v_{F}(x)=0$, where $v_{F}$ is the valuation of $k(x, y)$ corresponding to $F(x, y)$. Hence $v_{F}$ is trivial on $k(x)$. Any valuation of $k(x, y)=k(x)(y)$ trivial on $k(x)$ is of one of the following types: it is either the valuation corresponding to the infinite point, or the valuation corresponding to a monic irreducible polynomial from $k[x](y)$.

Since $F(x, y)=\pi(F(x, y)) a_{n}(x)$, we have $v_{F}(\pi(F(x, y)))=1$, hence $v_{F}$ coincides with the valuation of $k(x)(y)$ corresponding to $\pi(F(x, y))$. The lemma is proved.

We have $k\left(\mathbb{P}_{k}^{2}\right)=k(x, y)=k(x)(y)=k\left(\mathbb{P}_{k(x)}^{1}\right)$.

Corollary A 3. Let $\mathcal{A}$ be a central simple algebra over $k(x, y)$. Then $\mathcal{A} / k\left(\mathbb{P}_{k}^{2}\right)$ ramifies at $F(x, y)$ if and only if $\mathcal{A} / k\left(\mathbb{P}_{k(x)}^{1}\right)$ ramifies at $\pi(F(x, y))$. 
Proof. An algebra $\mathcal{A} / k\left(\mathbb{P}_{k}^{2}\right)$ ramifies at $F(x, y)$ if $\partial_{R_{F}}([\mathcal{A}]) \neq 0$, where $R_{F}$ is the valuation ring corresponding to the curve given by $F(x, y)=0$. Similarly, $\mathcal{A} / k\left(\mathbb{P}_{k(x)}^{1}\right)$ is ramified if $\partial_{R_{f}}([\mathcal{A}]) \neq 0$, where $R_{f}$ is the valuation ring corresponding to the polynomial $\pi(F(x, y))$. Since the valuations coincide, we have $R_{F}=R_{f}$, and the ramification maps coincide: $\partial_{R_{F}}=\partial_{R_{f}}$.

Since the valuations of $k(x, y)$ corresponding to $F(x, y)$ and $\pi(F(x, y))$ coincide, the corresponding ramification maps coincide as well. The corollary is proved.

Note that an algebra $\mathcal{A}$, viewed as an algebra over $k(x)\left(\mathbb{P}_{k(x)}^{1}\right)=k(x)(y)$, may ramify at the infinite point even if $\mathcal{A}$, viewed as an algebra over $k\left(\mathbb{P}_{k}^{2}\right)$, does not ramify at the infinite line $L_{\infty}$ given by $Z=0$. For example, consider a quaternion algebra $\mathcal{B}=\left(x y, x^{2}+1\right)$. It does not ramify at the infinite line because the valuation $v_{L_{\infty}}$ (corresponding to the infinite line) of both $x y$ and $x^{2}+1$ equals -2 . Indeed, $v_{L_{\infty}}$ coincides with the valuation $v_{\infty}$ of $k(x / y)(x)$ corresponding to the infinite point (cf. [18, p. 725]). Hence we have

$$
v_{L_{\infty}}(x y)=v_{\infty}(x y)=v_{\infty}\left(x^{2} y / x\right)=-2
$$

since $y / x$ lies in the "constant field" $k(x / y)$. Similarly, $v_{L_{\infty}}\left(x^{2}+1\right)=v_{\infty}\left(x^{2}+1\right)=$ -2 .

On the other hand, as an algebra over $k(x)\left(\mathbb{P}_{k(x)}^{1}\right), \mathcal{B}$ ramifies at the infinite point because $x^{2}+1 \notin k(x)^{2}$.

We regard the ramification locus of an algebra $\mathcal{A} / k\left(\mathbb{P}_{k}^{2}\right)$ as a divisor in $\mathbb{P}_{k}^{2}$ (maybe reducible).

We have the following

Lemma A 4 ([34, Lemma 1.1]). Let $R$ be a divisor in $\mathbb{P}_{k}^{2}$ corresponding to the ramification locus of an algebra $\mathcal{A} / k\left(\mathbb{P}_{k}^{2}\right)$. Then:

(1) $\operatorname{deg} R \geq 3$.

(2) If $\operatorname{deg} R=3$, then there are the following possibilities:

(a) $R$ is a union of three lines not passing through one point;

(b) $R$ is a union of a line and a conic not tangent to one another;

(c) $R$ is a nodal elliptic curve;

(d) $R$ is a smooth elliptic curve.

Ford [14] and Van den Bergh 34] proved that any algebra over $k(x, y)$ (where $k$ is algebraically closed) ramified only along a divisor of degree 3 is Brauer equivalent to a cyclic algebra. In the paper of Jacob [18 this assertion is generalized to the case of ramification along a hyperelliptic curve.

From our Propositions 2.2 and 2.3 it follows that any algebra with ramification divisor of degree 3 is Brauer equivalent to a cyclic algebra. Moreover, any algebra with ramification along a hyperelliptic curve is also Brauer equivalent to a cyclic algebra. The corresponding cyclic algebras can be exhibited explicitly.

Theorem A 5. Let $k$ be an algebraically closed field of characteristic zero, and let $\mathcal{A}$ be an algebra over $k(x, y)$ with ramification along a curve defined by an equation $y^{2}=h(x)$, where $h \notin k[x]^{2}$. Then $\mathcal{A}$ is Brauer equivalent to a cyclic algebra.

Proof. As an algebra over $k(x)(y), \mathcal{A}$ can be ramified only at the polynomial $y^{2}-h(x)$ and maybe at the infinite point. Moreover, since the ramification maps corresponding to $F(x, y)$ and $\pi(F(x, y))$ coincide, the ramification of $\mathcal{A} / k\left(\mathbb{P}_{k(x)}^{1}\right)$ at $\pi(F(x, y))$ is given by the same element in the residue field of the valuation as the 
ramification of $\mathcal{A} / k\left(\mathbb{P}_{k}^{2}\right)$ at $F(x, y)$. The ramification of $\mathcal{A} / k\left(\mathbb{P}_{k(x)}^{1}\right)$ at infinity is uniquely determined by the reciprocity law.

Since the degree of $y^{2}-h(x)$ with respect to $y$ is at most 2, Proposition 2.3 gives a cyclic algebra Faddeev equivalent to $\mathcal{A}$, and thus Brauer equivalent to this cyclic algebra. The theorem is proved.

Using Propositions 2.2 and 2.3, one can also prove the cyclicity of the algebras ramified at three lines or at a line and a conic (i.e. all cases of Lemma $\mathrm{A} 4$ are covered) 3

Theorem A 6. Let $k$ be an algebraically closed field of characteristic zero, and let $\mathcal{A}$ be an algebra over $k(x, y)$ with ramification at three lines

$$
a_{i} x+b_{i} y+c_{i}=0, i=1,2,3,
$$

or at a line $a x+b y+c=0$ and a conic $y^{2}=h(x), \operatorname{deg} h \leq 2$. Then $\mathcal{A}$ is Brauer equivalent to a cyclic algebra.

Proof. Let $\mathcal{A}$ be ramified at three lines. If $b_{1} \neq 0$, let $z=a_{1} x+b_{1} y$. Then $k(x, y)=k(x, z)$ and $\mathcal{A}$ is ramified at the lines $z+c_{1}=0, s_{i} x+t_{i} z+q_{i}=0$, $i=1,2$. Hence $\mathcal{A} / k\left(\mathbb{P}_{k(z)}^{1}\right)$ ramifies at two linear polynomials and maybe at the infinite point. From Proposition 2.2 we get an explicit form of a cyclic algebra. If $b_{1}=0, \mathcal{A} / k\left(\mathbb{P}_{k(x)}^{1}\right)$ ramifies at two linear polynomials and maybe at the infinite point, and we can apply Proposition 2.3, again noting that Faddeev equivalence coincides with Brauer equivalence.

Let $\mathcal{A}$ be ramified at a line $a x+b y+c=0$ and a conic $y^{2}=h(x)$. If $a \neq 0$, set $a x+b y=z$. Then $\mathcal{A}$ ramifies at the line $z+c=0$ and the conic $y^{2}=h((z-b y) / a)$. Hence $\mathcal{A} / k\left(\mathbb{P}_{k(z)}^{1}\right)$ ramifies at a quadratic polynomial and maybe at the infinite point. Proposition 2.3 gives an explicit form of a cyclic algebra Faddeev equivalent to $\mathcal{A}$. Finally, if $a=0, \mathcal{A} / k\left(\mathbb{P}_{k(y)}^{1}\right)$ ramifies at a quadratic polynomial and maybe at the infinite point, and we again apply Proposition 2.3 .

\section{ACKNOWLEDGEMENTS}

Yanchevskiu is grateful to Bar-Ilan University and Ben-Gurion University of the Negev (a substantial part of this work was done during his visits to BIU in 1996 and 2000 and to BGU in 2000). The work was continued while Kunyavskiı̌, Tikhonov, and Yanchevskil were visiting the University of Bielefeld, whose hospitality and support are also gratefully appreciated. We thank I. Efrat for useful discussions. We thank the referee whose critical remarks and suggestions were very helpful.

\section{REFERENCES}

[1] A. A. Albert, On the Wedderburn norm condition for cyclic algebras, Bull. Amer. Math. Soc. 37 (1931), 301-312.

[2] Algebraic Number Theory (J. W. S. Cassels and A. Fröhlich, Eds.), Academic Press, LondonNew York, 1967. MR0215665(35:6500)

[3] M. Artin, Left ideals in maximal orders, "Brauer Groups in Ring Theory and Algebraic Geometry", Lecture Notes Math. 917 (1982), 182-193. MR0657429 (83j:16009)

[4] M. Auslander and A. Brumer, Brauer groups of discrete valuation rings, Nederl. Akad. Wetensch. Proc. Ser. A 71 (1968), 286-296. MR0228471 (37:4051)

[5] S. Bloch, Torsion algebraic cycles, $K_{2}$, and Brauer groups of function fields, "Groupe de Brauer", Lecture Notes Math. 844 (1981), 75-102. MR0611866 (82k:14018)

\footnotetext{
${ }^{3}$ Added in proof. See our paper mentioned in footnote 1 for more general results.
} 
[6] E. S. Brussel, An arithmetic obstruction to division algebra decomposability, Proc. Amer. Math. Soc. 128 (2000), 2281-2285. MR.1662237(2000k:16018)

[7] J.-L. Colliot-Thélène (with an appendix by O. Gabber), Exposant et indice d'algèbres centrales simples non ramifiées, Enseign. Math. 48 (2002), 127-146. MR.1923420 (2003j:16023)

[8] J.-L. Colliot-Thélène and J.-J. Sansuc, On the Chow group of certain rational surfaces: a sequel to a paper of S. Bloch, Duke Math. J. 48 (1981), 421-447. MR0620258 (83e:14007)

[9] J.-L. Colliot-Thélène and Sir P. Swinnerton-Dyer, Hasse principle and weak approximation for pencils of Severi-Brauer and similar varieties, J. Reine Angew. Math. 453 (1994), 49112. MR:1285781 (95h:11060)

[10] R. Elman and T. Y. Lam, Quadratic forms and the u-invariant, II, Invent. Math. 21 (1973), 125-137. MR0417053 (54:5114)

[11] D. K. Faddeev, Simple algebras over a function field in one variable, Proc. Steklov Inst. 38 (1951), 321-344; English transl. in AMS Transl. 3 (1956), 15-38. MR0077505 (17:1046e)

[12] B. Fein and M. Schacher, Ulm invariant of the Brauer group of a field, Math. Z. 154 (1977), 41-50. MR0457410 (56:15615)

[13] B. Fein and M. Schacher, Brauer groups and character groups of function fields, J. Algebra 61 (1979), 249-255. MR0554861 (81c:12036)

[14] T. J. Ford, Division algebras that ramify along a singular plane cubic curve, New York J. Math. 1 (1995), 178-183. MR 1348596 (96i:12002)

[15] E. Frossard, Fibres dégénérées des schémas de Severi-Brauer d'ordres, J. Algebra 198 (1997), 362-387. MR.1489903 (99b:14006)

[16] V. I. Iskovskih, Rational surfaces with a pencil of rational curves, Mat. Sbornik 74 (116) (1967), 608-638; English transl. in Math. USSR Sb. 3 (1967), 563-587. MR0220734 (36:3786)

[17] V. I. Iskovskih, Minimal models of rational surfaces over arbitrary fields, Izv. Akad. Nauk SSSR Ser. Mat. 43 (1979), 19-43; English transl. in Math. USSR Izv. 14 (1980), 17-39. MR0525940 (80m:14021)

[18] B. Jacob, Division algebras which only ramify along a hyperelliptic curve in $\mathbb{P}^{2}$, J. Algebra 242 (2001), 720-728. MR.1848967 (2002f:16042)

[19] B. Jacob and A. Wadsworth, Division algebras over Henselian fields, J. Algebra 128 (1990), 126-179. MR.1031915 (91d:12006)

[20] A. Kresch, Hodge-theoretic obstruction to the existence of quaternion algebras, Bull. London Math. Soc. 35 (2003), 109-116. MR1934439 (2004c:16029)

[21] B. È. Kunyavskiı̌, A. N. Skorobogatov, and M. A. Tsfasman, Del Pezzo surfaces of degree four, Mem. Soc. Math. France, no. 37, Suppl. au Bull. Soc. Math. France 117 (1989), 1-113. MR:1016354 (90k:14035)

[22] B. E. Kunyavskiı̌ and M. A. Tsfasman, Zero-cycles on rational surfaces and Néron-Severi tori, Izv. Akad. Nauk SSSR Ser. Mat. 48 (1984), 631-654; English transl. in Math. USSR Izv. 24 (1985), 583-603. MR0747255 (86f:14004)

[23] T. Y. Lam, Algebraic Theory of Quadratic Forms, Benjamin, Reading, Mass., 1973. MR0396410 (53:277)

[24] S. Lang and A. Weil, Number of points of varieties in finite fields, Amer. J. Math. 76 (1954), 819-827. MR0065218 (16:398d)

[25] Yu. I. Manin, Cubic Forms: Algebra, Geometry, Arithmetic, Nauka, Moscow, 1972; English transl., 2nd ed., North-Holland, Amsterdam, 1986. MR0833513 (87d:11037)

[26] P. Morandi, The henselization of a valued division algebra, J. Algebra 122 (1989), 232-243. MR0994945 (90h:12007)

[27] U. Rehmann, S. V. Tikhonov, and V. I. Yanchevskii, Two-torsion of the Brauer groups of hyperelliptic curves and unramified algebras over their function fields, Comm. Algebra 29 (2001), 3971-3987. MR1857024(2002h:14030)

[28] S. Rosset and J. Tate, A reciprocity law for $K_{2}$-traces, Comment. Math. Helv. 58 (1983), 38-47. MR0699005 (85b:11105)

[29] D. Saltman, Division algebras over p-adic curves, J. Ramanujan Math. Soc. 12 (1997), 25-47; 13 (1998), 125-129. MR1462850 (98d:16032)

[30] J.-P. Serre, Corps locaux, 3ème éd., Hermann, Paris, 1968. MR0354618 (50:7096)

[31] J.-P. Serre, Cohomologie galoisienne, 5ème éd., Lecture Notes Math. 5, Springer-Verlag, Berlin et al., 1994. MR:1324577(96b:12010) 
[32] S. V. Tikhonov and V. I. Yanchevskiǔ, Unramified algebras of exponent 2 over function fields of split hyperelliptic curves, Dokl. Nats. Akad. Nauk Belarusi 45 (2001), no. 1, 42-45. MR.1850029

[33] S. V. Tikhonov and V. I. Yanchevskiū, Two-torsion of the unramified Brauer group of function fields of split hyperelliptic curves, Dokl. Nats. Akad. Nauk Belarusi 45 (2001), no. 3, 5-6. MR.1890418

[34] M. Van den Bergh, Division algebras on $\mathbb{P}^{2}$ of odd index ramified along a smooth elliptic curve are cyclic, Algèbre non commutative, groupes quantiques et invariants (Reims, 1995), Sémin. Congr., vol. 2, Soc. Math. France, Paris, 1997, pp. 43-53. MR1601190 (99d:16021)

[35] E. Witt, Schiefkörper über diskret bewerteten Körpern, J. Reine Angew. Math. 176 (1936), $153-156$

[36] V. I. Yanchevskiı̌ and G. L. Margolin, Brauer groups of local hyperelliptic curves with good reduction, Algebra i Analiz 7 (1995), no. 6, 227-249; 8 (1996), $n^{\circ} 1,237$; English transl. in St. Petersburg Math. J. 7 (1996), 1033-1048. MR1381984 (97c:11066a)

Department of Mathematics, Bar-Ilan University, 52900 Ramat Gan, Israel

E-mail address: kunyav@macs.biu.ac.il

Department of Mathematics, Bar-Ilan University, 52900 Ramat Gan, Israel

E-mail address: rowen@macs.biu.ac.il

Institute of Mathematics of the National Academy of Sciences of Belarus, ul. Surganova 11, 220072 Minsk, Belarus

E-mail address: tsv@im.bas-net.by

Institute of Mathematics of the National Academy of Sciences of Belarus, ul. Surganova 11, 220072 Minsk, Belarus

E-mail address: yanch@im.bas-net.by 